\title{
Multiangle Reconstruction of Domain Morphology with All-Optical Diamond Magnetometry
}

\author{
Lucio Stefan, ${ }^{1,2, *,+, \S}$ Anthony K.C. Tan $\odot,{ }^{1,+}$ Baptiste Vindolet, ${ }^{3,+}$ Michael Högen, ${ }^{1}$ Dickson Thian $\odot,{ }^{4}$ \\ Hang Khume Tan $\oplus,{ }^{4}$ Loïc Rondin $\odot,{ }^{3}$ Helena S. Knowles $\odot,{ }^{1}$ Jean-François Roch, ${ }^{3}$ \\ Anjan Soumyanarayanan $\odot,{ }^{4,5}$ and Mete Atatüre $\odot^{1, \dagger}$ \\ 'Cavendish Laboratory, University of Cambridge, J. J. Thomson Avenue, Cambridge CB3 OHE, United Kingdom \\ ${ }^{2}$ The Faraday Institution, Quad One, Becquerel Avenue, Harwell Campus, Didcot OX11 ORA, United Kingdom \\ ${ }^{3}$ Université Paris-Saclay, CNRS, ENS Paris-Saclay, CentraleSupélec, LuMIn, Gif-sur-Yvette 91190, France \\ ${ }^{4}$ Institute of Materials Research and Engineering, Agency for Science, Technology and Research (A*STAR), \\ 138634, Singapore \\ ${ }^{5}$ Physics Department, National University of Singapore (NUS), 117551, Singapore
}

(Received 22 January 2021; revised 10 June 2021; accepted 17 June 2021; published 22 July 2021)

\begin{abstract}
Scanning diamond magnetometers based on the optically detected magnetic resonance of the nitrogenvacancy center offer very high sensitivity and noninvasive imaging capabilities when the stray fields emanating from ultrathin magnetic materials are sufficiently low (less than $10 \mathrm{mT}$ ). Beyond this low-field regime, the optical signal quenches and a quantitative measurement is challenging. While the fielddependent photoluminescence from the nitrogen-vacancy center can still provide qualitative information on magnetic morphology, this operation regime remains unexplored, particularly for surface magnetization larger than approximately $3 \mathrm{~mA}$. Here, we introduce a multiangle reconstruction (MARE) that captures the full nanoscale domain morphology in all magnetic field regimes leading to photoluminescence quench. To demonstrate this, we use $[\mathrm{Ir} / \mathrm{Co} / \mathrm{Pt}]_{14}$ multilayer films with surface magnetization an order of magnitude larger than previous reports. Our approach brings noninvasive nanoscale magnetic field imaging capability of the nitrogen-vacancy center to the study of a wider pool of magnetic materials and phenomena.
\end{abstract}

DOI: 10.1103/PhysRevApplied.16.014054

\section{INTRODUCTION}

The negatively charged nitrogen-vacancy center (NV) in diamond has attracted great interest as a versatile quantum sensor for the investigation of weak-field magnetism that demands high sensitivity, nanoscale resolution, and noninvasiveness [1-5]. In the presence of a magnetic field, the Zeeman splitting of the NV spin can be quantified by performing optically detected magnetic resonance (ODMR) measurements using laser and microwave excitation [6]. The single-spin nature of the NV also ensures limited perturbation of the measured system. Furthermore, attaching a NV-containing diamond platform on a scanning probe [2,3,7-9] enables scanning NV microscopy (SNVM), which allows for nanoscale noninvasive magnetic imaging. This technique features a large operating temperature range (cryogenic to room temperature) and

\footnotetext{
*1ucio.stefan@nbi.ku.dk

†ma424@cam.ac.uk

$\$$ These authors contributed equally to this work.

${ }^{\S}$ Current address: Niels Bohr Institute, University of Copenhagen, Blegdamsvej 17, 2100 Copenhagen, Denmark.
}

stability in vacuum to ambient conditions $[1,6,10]$. However, the ODMR measurements are restricted to magnetic fields below $10 \mathrm{mT}$ due to the field-induced quenching of the ODMR contrast, thus preventing the optical readout of the spin splitting $[8,11,12]$. As a consequence, quantitative ODMR-based SNVM has been demonstrated mainly on magnetic textures in thin films with close to zero surface magnetization, such as antiferromagnetic or single-layer ferromagnetic materials [4,7,8,11,13-20].

To extend the operational range beyond $10 \mathrm{mT}$, the $\mathrm{NV}$ can harness the field-dependent quench of the NV photoluminescence (PL) for magnetic imaging, as demonstrated recently [11,21-23]. Quench-based SNVM monitors the changes in NV PL due to the local magnetic field variation across a spin texture with respect to the NV quantization axis. This modality also offers reduced acquisition time and enables microwave-free nonperturbative operation $[5,24,25]$. The interpretation of quench-based SNVM maps can be ambiguous, because of the multiple parameters that influence PL quenching, such as the NV-sample distance, NV axis orientation, sample magnetization, magnetic domain size, or magnetic field noise [26]. Therefore, this imaging mode has been limited to the mapping of 
magnetic domain morphology with surface magnetization $I_{S} \lesssim 3 \mathrm{~mA}$ [21-23] (equivalent to $2 \mathrm{~nm}$ of Co). In this report, we reveal distinct quench-based imaging regimes, dependent on the material parameters, and introduce the Multiangle Reconstruction (MARE) protocol to interpret the domain morphology from quenched SNVM maps. We demonstrate MARE on an $[\mathrm{Ir} / \mathrm{Co} / \mathrm{Pt}]_{14}$ multilayer film with $12 \mathrm{~mA}$ out-of-plane surface magnetization, an order of magnitude larger than the operational limit of ODMRbased SNVM. Utilizing MARE can extend the applicability of SNVM to a wider range of materials and magnetic regimes.

\section{QUENCH-BASED IMAGING IN DIFFERENT REGIMES}

Figure 1(a) illustrates our experimental setup consisting of a diamond scanning probe with a NV implanted close to the diamond surface at a NV-sample distance $d_{\mathrm{NV}}$ smaller than $100 \mathrm{~nm}[2,9,27,28]$. The optical ground state of the $\mathrm{NV}$ is a spin triplet, with a quantization axis $\hat{u}_{\mathrm{NV}}$ along one of the four crystallographic axes of the diamond lattice $[29,30]$ and the lowest-energy state $\left|m_{s}=0\right\rangle$ is split from the $\left|m_{s}= \pm 1\right\rangle$ states by $2.87 \mathrm{GHz}[31]$. The local magnetic field can be decomposed into parallel $\left(\mathbf{B}_{\|}\right)$and orthogonal $\left(\mathbf{B}_{\perp}\right)$ components with respect to $\hat{u}_{\mathrm{NV}}$ [see the inset of Fig. 1(a)]. The $\mathbf{B}_{\|}$component splits the $\left|m_{s}= \pm 1\right\rangle$ states that are measured by monitoring the ODMR [32]. However, $\mathbf{B}_{\perp}$ changes the eigenbasis and modifies the branching ratio of the optical transitions [12]. This results in the quenching of the NV PL and the suppression of the ODMR contrast (Appendix A), restricting quantitative ODMR-based imaging to below approximately $10 \mathrm{mT}$ [11,12].

Quench-based SNVM generates a PL intensity map, where regions with strong $\mathbf{B}_{\perp}$ component appear darker. In the limit of modest surface magnetization and small NVsample distance $d_{\mathrm{NV}}$, the domain boundaries appear dark, producing faithful magnetic domain morphology maps. Therefore, demonstrations are limited to single- or bilayer thin-film systems with surface magnetization $I_{s} \lesssim 3 \mathrm{~mA}$ [8,11,21-23]. Outside this regime, the complex interplay between $d_{\mathrm{NV}}$ and $I_{s}$, as well as the morphology length scale, on the NV PL obfuscates the straightforward correspondence of dark regions to domain boundaries. Therefore, a systematic understanding of quench-based SNVM response is necessary to retrieve the domain morphology of a magnetic material. To do this, we first simulate the $d_{\mathrm{NV}}$ dependence of quench-based SNVM for a known magnetic structure.

Our study involves the $[\operatorname{Ir}(1 \mathrm{~nm}) / \operatorname{Co}(1) / \operatorname{Pt}(1)]_{14}$ magnetic multilayer, a room-temperature skyrmion platform with an out-of-plane anisotropy, and $I_{s}=12 \mathrm{~mA}$ (Appendix B) - an order of magnitude larger than systems studied previously with SNVM. Furthermore, the ambient
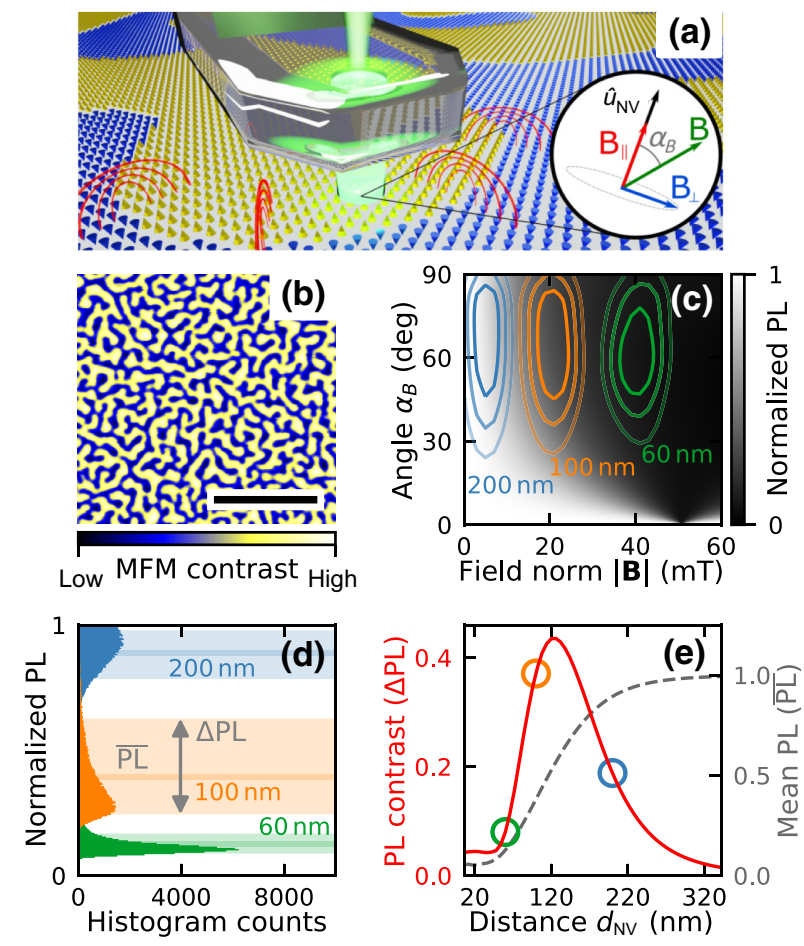

FIG. 1. Effect of magnetic field amplitude and orientation on NV luminescence. (a) Illustration of a diamond probe scanning over a spin texture (colored cones) with magnetic field lines across the domain boundaries (red lines). The inset is a schematic of the local magnetic field vector $\mathbf{B}$ with reference to the $\mathrm{NV}$ quantization axis $\hat{u}_{\mathrm{NV}}$ at the tip of the diamond probe. Here $\alpha_{B}$ indicates the angle between $\mathbf{B}$ and $\hat{u}_{\mathrm{NV}}$. (b) Labyrinth domain morphology in an Ir/Co/Pt multilayer observed by MFM, exhibiting a zero-field period of $407 \mathrm{~nm}$ (scale bar is $3 \mu \mathrm{m}$ ). (c) Normalized NV luminescence defined as $\left(\mathrm{PL}-\mathrm{PL}_{\min }\right) /\left(\mathrm{PL}_{\max }-\right.$ $\left.\mathrm{PL}_{\text {min }}\right)$ as a function of $|\mathbf{B}|$ and $\alpha_{B}$. The contour lines of the $|\mathbf{B}|-$ $\alpha_{B}$ two-dimensional histograms at three selected $d_{\mathrm{NV}}(60,100$, and $200 \mathrm{~nm}$ ), obtained from the simulated magnetic field across (b), are overlaid on (c) (green, orange, and blue contour lines). The 80th, 60th, and 40th percentiles of each distribution are indicated with increasingly lighter contour lines. (d) The histograms of the simulated PL response at the three $d_{\mathrm{NV}}$ values 60,100 , and $200 \mathrm{~nm} . \overline{\mathrm{PL}}$ indicates the mean PL, $\Delta \mathrm{PL}$ marks the difference between the 90th and 10th percentiles of the PL distribution. (e) Dependence of $\Delta \mathrm{PL}$ and $\overline{\mathrm{PL}}$ on $d_{\mathrm{NV}}$. The peak of $\Delta \mathrm{PL}$ marks the optimal distance for quench-based imaging for the multilayer film of (b). The colored circles correspond to the three $d_{\mathrm{NV}}$ values considered in panels (c) and (d).

stability of the nanoscale spin textures $[33,34]$ allows us to correlate the quench-based SNVM images with magnetic force microscopy (MFM) measurements [35]. Figure 1(b) presents a MFM image of this film, exhibiting a labyrinth domain morphology with a zero-field period of $407 \mathrm{~nm}$. Figure 1(c) presents a gray-scale map of normalized PL intensity simulated as a function of field amplitude $|\mathbf{B}|$ and field angle $\alpha_{B}$ with respect to the NV axis $\hat{u}_{\mathrm{NV}}$. To understand how the stray field distribution of the domain 
morphology affects the NV PL at various $d_{\mathrm{NV}}$, we simulate the volumetric field distribution from the MFM map in Fig. 1(b) using the micromagnetics package $\operatorname{MUMAX}^{3}$ [36] (Appendix C). On Fig. 1(c), we overlay the corresponding $|\mathbf{B}|-\alpha_{B}$ distributions of the magnetic field at three different $d_{\mathrm{NV}}$ : $60 \mathrm{~nm}$ (green contours), $100 \mathrm{~nm}$ (orange), and 200 $\mathrm{nm}$ (blue) (Appendix D). At $d_{\mathrm{NV}}=60 \mathrm{~nm}(200 \mathrm{~nm})$, the NV PL remains uniformly quenched (unaffected) for the majority of the field distribution, while $100 \mathrm{~nm} d_{\mathrm{NV}}$ results in strong PL variation. Figure 1(d) clearly highlights these NV PL variations $\triangle \mathrm{PL}$ via the corresponding histograms at $d_{\mathrm{NV}}=60,100$, and $200 \mathrm{~nm}$. Figure 1(e) presents the $\triangle \mathrm{PL}$ - calculated as the difference between the 90th and 10th percentiles of the NV PL distribution-as a function of $d_{\mathrm{NV}}$ (solid red curve) alongside the mean PL (dashed gray curve).

To assess the operational regime of quench-based SNVM, we need to consider further the interplay between $I_{s}$ and $d_{\mathrm{NV}}$. As shown in Fig. 2(a), quench-based SNVM can be categorized into different regimes. The combination of large $d_{\mathrm{NV}}$ and small $I_{s}$ (small $d_{\mathrm{NV}}$ and large $I_{s}$ ) results in predominantly bright (quenched) PL maps. In both the no quench and the full quench regimes, the lack of PL variation $\triangle \mathrm{PL}$ implies that little to no morphological information of the underlying spin textures is captured. In contrast, quench-based SNVM is feasible in the partial quench regime [area bounded by the dotted lines in Fig. 2(a)] for a limited range of $I_{s}$ and $d_{\mathrm{NV}}$ combinations. While the partial quench regime gives a large $\Delta \mathrm{PL}$, which is desirable for quench-based SNVM, the resultant PL maps over an identical spin texture can vary dramatically across this regime. To highlight this, we simulated quench-based SNVM maps of the same area in the multilayer film using three different combinations of $I_{s}$ and $d_{\mathrm{NV}}$ [Figs. 2(b)-2(d)]. In general, we observe an evolution from dark, isotropic features at lower $I_{S}$ and $d_{\mathrm{NV}}$ to bright, directional features at higher $I_{s}$ and $d_{\mathrm{NV}}$ due to competing magnetic field contributions above domains and domain boundaries. At lower $I_{s}$ and $d_{\mathrm{NV}}$ [blue region in Fig. 2(a)], the quench image appears as a uniform bright background with isotropic dark
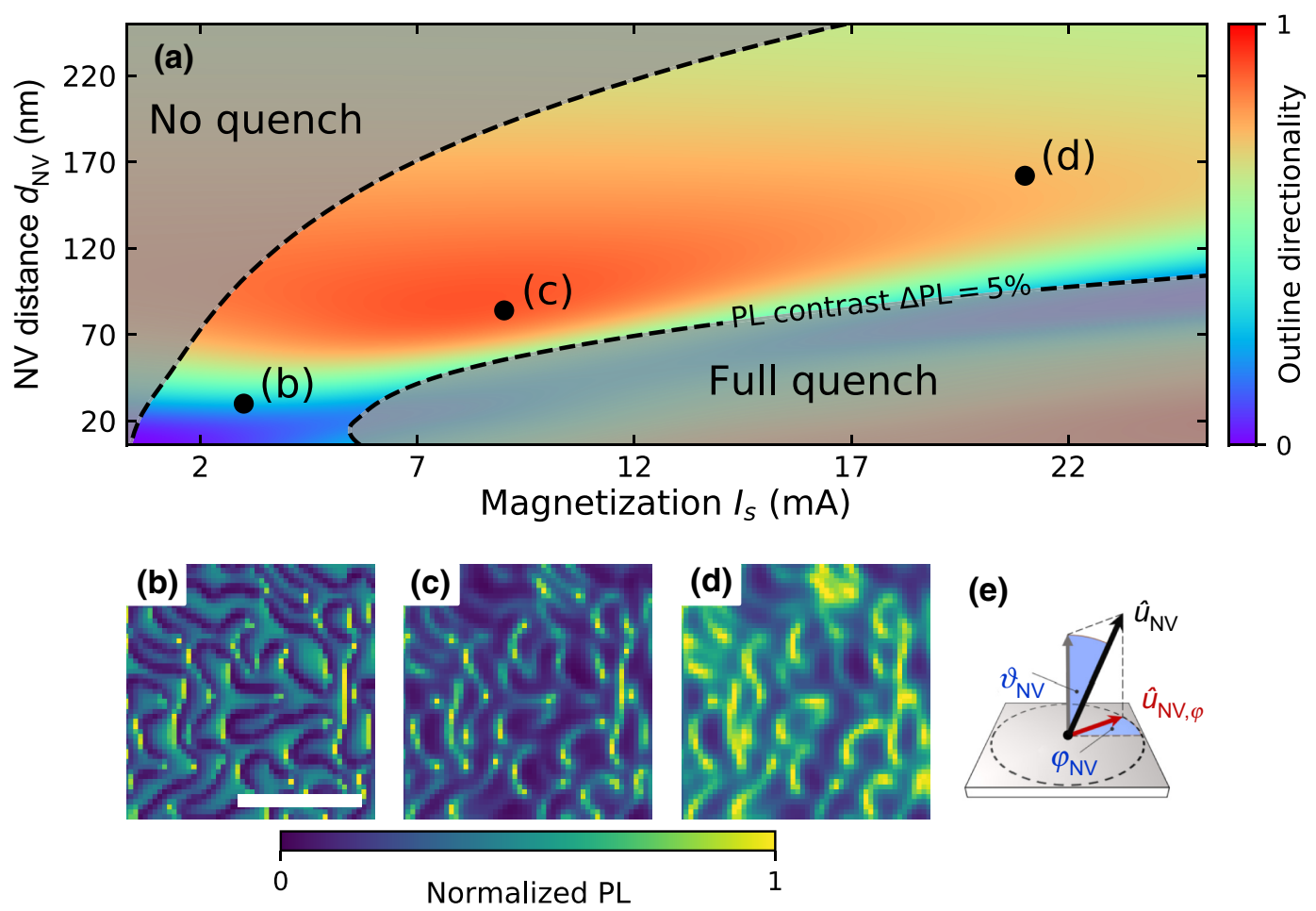

FIG. 2. Quench-based SNVM imaging regimes. (a) Different regimes of quench-based imaging as a function of the NV-sample distance $\left(d_{\mathrm{NV}}\right)$ and surface magnetization $\left(I_{s}\right)$, based on simulated quench images. Little to no domain morphological information is captured in the no quench (left grayed area) and full quench (right grayed area) regimes where the PL map is predominantly bright or dark, respectively. In the partial quench regime (area bounded by dashed lines), field variations are mapped to PL changes resulting in (b)-(d) quench images with features indicative of domain boundaries (scale bar is $1 \mu \mathrm{m}$ ). Domain boundaries appear as dark isotropic PL features (low directionality) for smaller $d_{\mathrm{NV}}$ and $I_{s}(\mathrm{~b})$, and as directional bright features (high directionality) at larger $d_{\mathrm{NV}}$ and $I_{s}$ (c),(d). The orientation of the directionality depends on $\hat{u}_{\mathrm{NV}, \varphi}$ which is the NV axis $\hat{u}_{\mathrm{NV}}$, projected on the sample surface. The dashed lines indicate the contour lines for 5\% map contrast. (e) Illustration depicting the NV axis $\hat{u}_{\mathrm{NV}}$, the tilt angle $\vartheta_{\mathrm{NV}}$ from the normal to the sample surface, and the projection of $\hat{u}_{\mathrm{NV}}$ onto the sample plane, $\hat{u}_{\mathrm{NV}, \varphi}$. The angle $\varphi_{\mathrm{NV}}$ is the angle between $\hat{u}_{\mathrm{NV}, \varphi}$ and the reference axis within the sample plane. For panels (a)-(d), $\vartheta_{\mathrm{NV}}=54.7^{\circ}$ and $\varphi_{\mathrm{NV}}=0^{\circ}$. 
outlines [Fig. 2(b)]. This is a result of the strong magnetic field localized at the domain boundaries that quenches the NV. The NV quench images reported to date lie in this region of the parameter space [8,21-23] (Appendix D).

For combinations of larger $I_{s}$ and $d_{\mathrm{NV}}$ values [orange region in Fig. 2(a)], the quench maps generate strikingly different images: panels (c) and (d) capture highly directional bright and segmented features along the domain boundaries. In this case, a strong off-axis magnetic field above domains and domain boundaries results in a predominantly dark PL map. However, due to large gradients localized at domain boundaries, there are instances where the field is aligned closer to $\hat{u}_{\mathrm{NV}}$. This occurs across portions of domain boundaries orthogonal to the projection of $\hat{u}_{\mathrm{NV}}$ in the sample plane $\left(\hat{u}_{\mathrm{NV}, \varphi}\right)$, resulting in directional bright features for panels (c) and (d), highly dependent on the NV equatorial angle $\varphi_{\mathrm{NV}}$ [Fig. 2(e)]. Notably, this directional behavior occurs over a significantly larger parameter space of the partial quench regime, well beyond that of panel (a), and the trend remains valid for different domain periodicities (Appendix D). It is worth emphasizing here that magnetic materials with $I_{s}$ larger than approximately $3 \mathrm{~mA}$ would inevitably constrain quench-based SNVM to the directional region of Fig. 2(a). Therefore, a protocol that relates these images with the actual magnetic domain morphology is necessary in order to extend the operation regime of quench-based SNVM for nonperturbative investigations of such materials. With the growing interest and recent development of (111)-oriented diamond probes [37] (i.e., $\vartheta_{\mathrm{NV}}=0^{\circ}$ ), we have also extended our simulation work to [111] NV for quench-based SNVM (see Appendix F).

\section{RECONSTRUCTION OF DOMAIN MORPHOLOGY-MARE}

To reflect the role of $\varphi_{\mathrm{NV}}$ in quench-based SNVM, we simulate two quench images for $\varphi_{\mathrm{NV}}=0^{\circ}$ and $\varphi_{\mathrm{NV}}=90^{\circ}$, displayed in Figs. 3(a) and 3(b), respectively. We set $d_{\mathrm{NV}}=77 \mathrm{~nm}, \vartheta_{\mathrm{NV}}=54.7^{\circ}$, and an approximately $12 \mathrm{~mA}$ surface magnetization (Appendix E) to reflect our experimental measurements. The images for both $\varphi_{\mathrm{NV}}$ orientations show directional segments revealing some features of the domain morphology, but more importantly these segments are complementary. Therefore, while an image at a given $\varphi_{\mathrm{NV}}$ remains incomplete, images obtained at multiple $\varphi_{\mathrm{NV}}$ values can collectively give a significantly better coverage of the underlying domain morphology, which is the essence of the proposed imaging protocol. The MARE protocol harnesses the $\varphi_{\mathrm{NV}}$ dependence of PL features to build a composite map enabling morphological imaging further into the partial quench regime, i.e., in strong-field conditions.

The overlapping features in the PL maps obtained at different $\varphi_{\mathrm{NV}}$, e.g., $0^{\circ}$ and $90^{\circ}$ as in panels (a) and (b) of
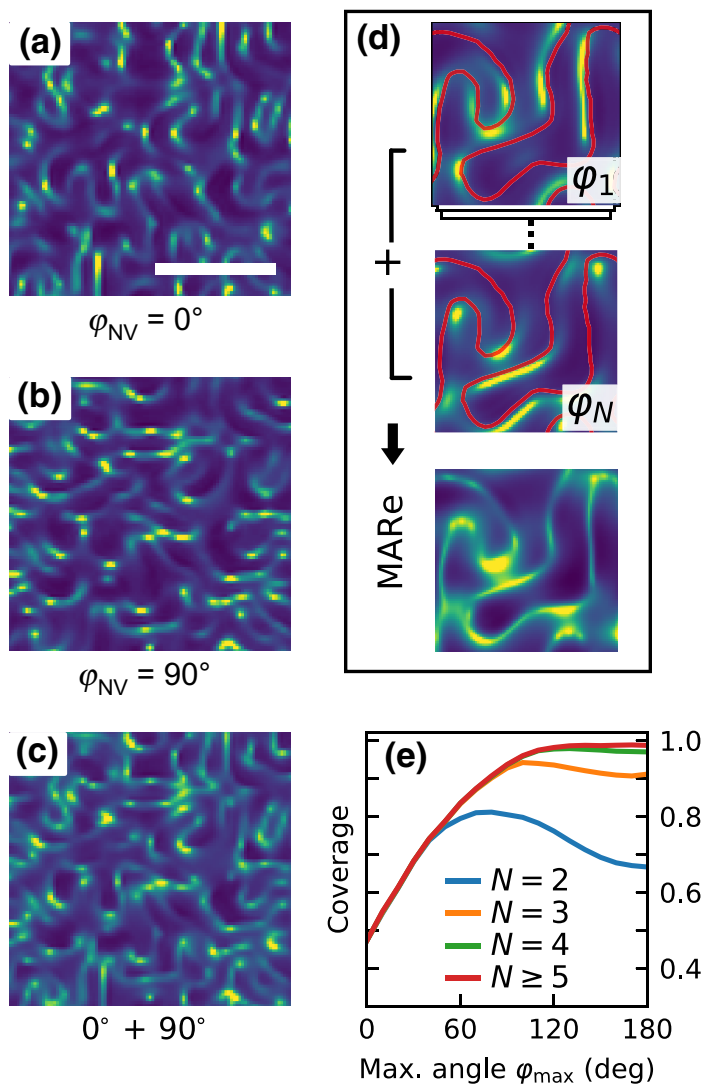

FIG. 3. Directional quench imaging and morphology reconstruction. (a) Simulated quenched PL map based on spin texture in Fig. 1(b), with $\vartheta_{\mathrm{NV}}=54.7^{\circ}, \varphi_{\mathrm{NV}}=0^{\circ}$, and (b) simulated quenched PL map in the same area but with the NV rotated $90^{\circ}$ in the sample plane $\left(\varphi_{\mathrm{NV}}=90^{\circ}\right)$. Both maps are simulated at a NVsample distance $d_{\mathrm{NV}}=77 \mathrm{~nm}$ and surface magnetization $I_{s}=$ $12 \mathrm{~mA}$ (scale bar is $1 \mu \mathrm{m}$ ). (c) Reconstructed image obtained by summing (a) and (b). (d) MARE illustrating the domain morphology acquisition based on multiple $N$ images at various $\varphi_{\mathrm{NV}}$. (e) Coverage of domain boundaries given as functions of $N$ and $\varphi_{\mathrm{NV}}{ }^{\max }$. Here $N$ is the number of quench images involved in the reconstruction, and are obtained over a range of $\varphi_{\mathrm{NV}}\left(0^{\circ}\right.$ to $\left.\varphi_{\mathrm{NV}}{ }^{\max }\right)$ spaced by $\Delta \varphi_{\mathrm{NV}}=\varphi_{\mathrm{NV}}^{\max } /(N-1)$. The reconstruction with $N=4$ images yields the largest coverage of approximately equal $98 \%$, which saturates above $\varphi_{\mathrm{NV}}{ }^{\max } \simeq 120^{\circ}$. A coverage of $100 \%$ indicates that all the domain boundaries are captured.

Fig. 3, allow us to perform an initial image registration without prior MFM imaging or any form of morphological information of the domains. This post process is to compensate for the domain outline shift caused by $\vartheta_{\mathrm{NV}} \neq 0^{\circ}$ (details in Sec. IV). Subsequently, the maps are normalized and summed to yield a MARE image, as displayed in Fig. 3(c), revealing a larger fraction of the domain boundaries with just two values of $\varphi_{\mathrm{NV}}$. To quantify the domain boundary coverage, we integrate the product of the domain outlines from the MFM image [Fig. 1(b)] with the binarized MARE image. In order to maximize the fraction of domain boundaries covered by the protocol, we 
consider $N \geq 2$ images taken at different $\varphi_{\mathrm{NV}}$ values ranging from $0^{\circ}$ to $\varphi_{\mathrm{NV}}{ }^{\max }$ spaced equally by $\Delta \varphi_{\mathrm{NV}}=\varphi_{\mathrm{NV}}^{\max } /$ $(N-1)$. Figure 3(d) illustrates the MARE scheme for $N=$ 4 and $\varphi_{\mathrm{NV}}{ }^{\max }=120^{\circ}$, which corresponds to four quenchbased SNVM images with each obtained at a $40^{\circ}$ relative angle. The corresponding MARE image clearly captures an increased fraction of the domain morphology.

Figure 3(e) presents the calculated fraction of domain boundary coverage for MARE with $N=2,3$, and 4 (black, blue, and red curves). For $N=2$ (3), the maximum coverage reaches $81 \%(94 \%)$ at $\varphi_{\mathrm{NV}}{ }^{\max }=80^{\circ}\left(100^{\circ}\right)$. Extending MARE to $N=4$ further improves the coverage, reaching a maximum of approximately $98 \%$. This shows that, even for $N \leq 4$, the MARE protocol is capable of recovering the domain morphology with near-unity coverage. We further explore the option of using more than four images $(N>4)$ [Fig. 3(e)], which results in a nearunity coverage. However, increasing the MARE protocol to $N>5$ does not result in an appreciable increase in coverage.

Figure 4 presents our experimental demonstration of domain morphology mapping using MARE on the $[\mathrm{Ir} / \mathrm{Co} / \mathrm{Pt}]_{14}$ multilayer. We perform quench-based SNVM using a custom scanning NV setup with commercial diamond probes. In this study, we obtain quench images by using a [100] diamond probe containing a single NV with $\vartheta_{\mathrm{NV}}=60^{\circ} \pm 2^{\circ}$ and $d_{\mathrm{NV}}=77 \pm 3 \mathrm{~nm}$ (Appendix E). The combination of the $d_{\mathrm{NV}}(77 \mathrm{~nm})$ value and $I_{s}(12 \mathrm{~mA})$ of the $[\mathrm{Ir} / \mathrm{Co} / \mathrm{Pt}]_{14}$ multilayer yields directional quench images according to Fig. 2(a). Figures 4(a) and 4(b) show experimental quench images acquired at $\varphi_{\mathrm{NV}}=0^{\circ}$ and $\varphi_{\mathrm{NV}}=90^{\circ}$, respectively, on the same area used for simulating Figs. 3(a) and 3(b) (Appendix B). The image at
$\varphi_{\mathrm{NV}}=90^{\circ}$ is obtained by rotating the sample with respect to the diamond probe, followed by an image rotation in postprocessing so that the image is presented in the same orientation as in Fig. 4(a). In addition to the image process required to obtain a MARE image, the experimental single quench images are preregistered to their respective simulated quench images to correct for nonlinear distortions caused by the open-loop piezoelectric scanners. The domain boundary coverage of each of these images is $60_{-11}^{+13 \%}$, in line with the simulations and there is good agreement between the simulated and the measured images for both orientations (Sec. IV). Figure 4(c) is the corresponding $N=2$ MARE image showing matching bright features with the highlighted domain boundaries of the binarized MFM image displayed in Fig. 4(d). The experimentally achieved domain boundary coverage is calculated to be $71_{-15}^{+12 \%}$ - an enhancement beyond the single frame coverage of approximately $60 \%$. The deviation from the simulated $N=2$ MARE coverage value of $81 \%$ could be due to a combination of factors, including nonlinearity of the experimental maps and nonideal image processing parameters (registration, binarization, edge detection; see Sec. IV and Appendix G). Another reason for this deviation might be due to perturbations of the domain morphology induced by MFM scanning. As the experimental protocol includes MFM scans performed before and after each quench-based SNVM map, we do observe local perturbations due to MFM that could potentially lead to deviations from the unperturbed images captured by quench-based SNVM (see Appendix H). Nonetheless, the experimental demonstration of MARE extends the operational range of noninvasive quench-based SNVM into the partial quench regime.
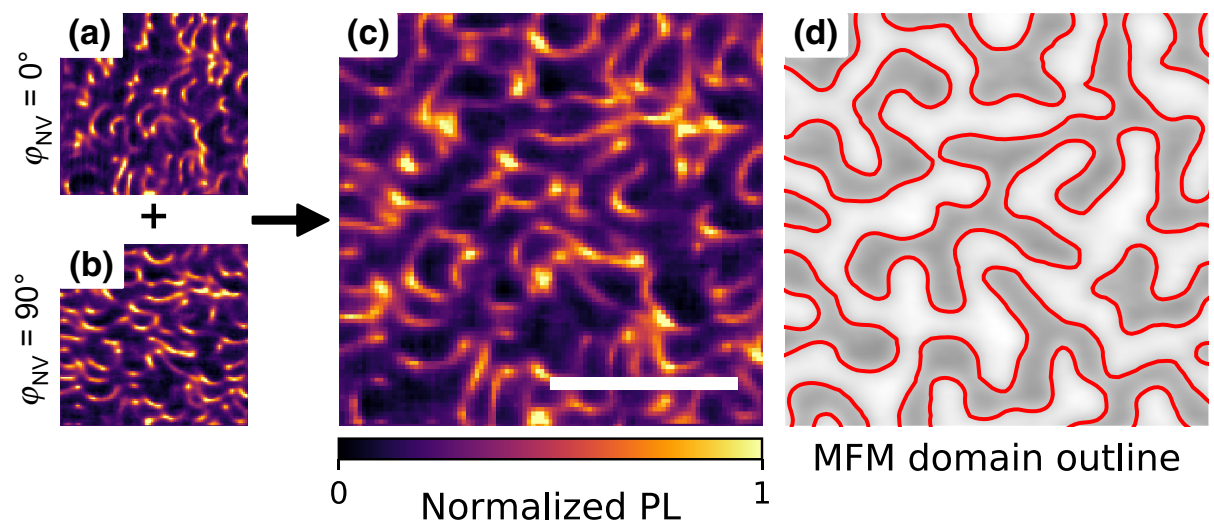

FIG. 4. Experimental verification of multiangle reconstruction of domain morphology. Experimental quenching map of the same areas as in Figs. 3(a) and 3(b) with $\vartheta_{\mathrm{NV}}=60^{\circ} \pm 2^{\circ}$ and (a) $\varphi_{\mathrm{NV}}=0^{\circ}$ and (b) $\varphi_{\mathrm{NV}}=90^{\circ}$. The change in $\varphi_{\mathrm{NV}}$ is achieved by rotating the sample by $90^{\circ}$ with reference to the diamond tip. The two images are combined to give (c) the experimental MARE image at $N=2$ (scale bar is $1 \mu \mathrm{m}$ ). The experimental coverage is calculated to be $71_{-15}^{+12} \%$ with reference to the domain boundary obtained from the MFM map over the same area in (d). (d) Cropped and gray scaled MFM map from Fig. 1(b), covering the same area as in (a)-(c). The illustration of the domain boundaries (in red) are lines interpolated from the pixelated boundaries of a binarized MFM image (see the details in Appendix G). 


\section{CROSS-CORRELATION ANALYSIS OF MARE IMAGES}

We further analyze the MARE protocol by studying the two-dimensional cross-correlation of single quench and MARE images with the domain boundaries. We first simulate two single quenched maps at $\varphi_{\mathrm{NV}}=0^{\circ}$ and $\varphi_{\mathrm{NV}}=$ $90^{\circ}\left(\vartheta_{\mathrm{NV}}=54.7^{\circ}\right)$; see Figs. 5(a) and 5(b). The crosscorrelation between the quench maps and the domain boundaries - obtained from the MFM map with the Canny edge detection algorithm - is shown in Figs. 5(d) and 5(e). In both images, a positive and elongated positive crosscorrelation peak is apparent. The peak is shifted from the origin and the orientation of the major axis is orthogonal to the orientation of $\hat{u}_{\mathrm{NV}, \varphi}$. The shift originates from the nonzero tilt of $\hat{u}_{\mathrm{NV}}$ from the normal to the sample plane
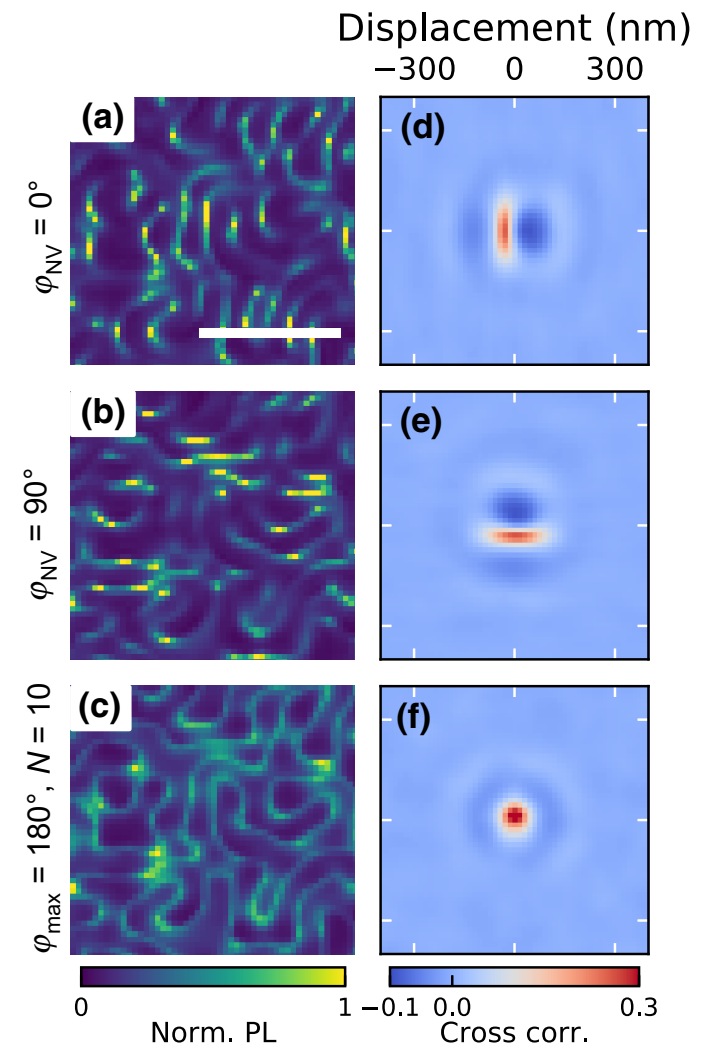

FIG. 5. Directionality and image reconstruction. (a),(b) Simulated quenched maps with $\varphi_{\mathrm{NV}}=0^{\circ}$ and $\varphi_{\mathrm{NV}}=90^{\circ}$ and (c) the MARE image with $N=10$ and $\varphi_{\mathrm{NV}}{ }^{\max }=180^{\circ}$ (scale bar is $1 \mu \mathrm{m})$. (d)-(f) Two-dimensional cross-correlations between the quenched images in (a)-(c) and the domain boundaries. For single images (d),(e), the cross-correlation shows a positive correlation shifted from the origin in the direction opposite to $\hat{u}_{\mathrm{NV}, \varphi}$, the projection of $\hat{u}_{\mathrm{NV}}$ in the sample plane. This indicates that the bright outlines are highly directional and do not occur exactly on top of the domain boundaries. On the contrary, the crosscorrelation of the MARE map (f) is isotropic, indicating that most of the boundaries are uniformly covered. and needs to be compensated with image registration algorithms before combining the images (Appendix G), while the $\varphi_{\mathrm{NV}}$ dependence of the peak highlights the directionality of the single quenched maps. In contrast, a map reconstructed with an $N=10$ MARE protocol [Fig. 5(c)] results in a rotationally symmetric positive cross-correlation peak [Fig. 5(f)]. The registration of the single quenched images to the domain boundaries, used to calculate the coverage as pointed out in Sec. III, also ensures that the peak occurs at zero shift from the origin.

\section{OUTLOOK}

Our work methodically evaluates quench-based SNVM in terms of characteristic NV and magnetic material properties. We establish a predictive scheme involving MFM, micromagnetics, and NV photodynamics simulations, which yields images in excellent agreement with experimentally acquired data. We find two regimes of quench imaging where morphological information is captured. The first regime corresponds to mostly bright PL maps with dark outlines tracing the domain boundaries, which correspond to materials of low magnetization $\left(I_{s} \lesssim\right.$ $3 \mathrm{~mA}$ ). The second regime, which has not been reported to date, results in PL maps with directional segmented features with strong $\hat{u}_{\mathrm{NV}, \varphi}$ dependence. We established a MARE scheme to enable domain morphology mapping with near-unity coverage for the second regime. The experimentally validated MARE protocol extends quench-based SNVM imaging of out-of-plane spin textures to magnetic systems with $I_{s} \gtrsim 3 \mathrm{~mA}$. Furthermore, the scheme to identify the imaging regimes can be generalized to complex magnetic textures, thus enabling the forecast of the attainable SNVM modes. While the protocol requires multiple quench-based images, it is still less time consuming than ODMR-based imaging. Moreover, the postprocessing is fast and can be automated, and ultimately brings SNVM to a previously inaccessible regime that has no other nonperturbative imaging alternatives. We posit that the nonperturbative nature of this technique will be increasingly attractive for research towards miniaturization. Operationally, the execution of the MARE protocol can be improved drastically in terms of time and effort, with closeloop positioning and/or a piezoelectric rotation stage, both of which are easily obtainable. We anticipate that these insights, alongside tools developed for prediction, interpretation, and reconstruction, will stimulate the adoption of quench-based SNVM as a nonperturbative nanoscale magnetometry to a wider pool of materials, thereby furthering the development of quantitative quench-based SNVM imaging.

\section{ACKNOWLEDGMENTS}

This work was performed at the Cambridge Nanoscale Sensing and Imaging Suite (CANSIS), part of the 
Cambridge Henry Royce Institute, under EPSRC Grant No. EP/P024947/1. We further acknowledge funding from EPSRC QUES2T (EP/N015118/1) and from the Betty and Gordon Moore Foundation. This work is also supported by the Faraday Institution (FIRG01) and by the SpOT-LITE programme (Grants No. A1818g0042 and No. A18A6b0057), funded by Singapore's RIE2020 Initiatives. A.K.C.T. acknowledges funding from A*STAR, Singapore. B.V. acknowledges support by a Ph.D. research grant from the Délégation Générale de l'Armement. H.S.K. acknowledges funding from the Royal Society through a Royal Society University Research Fellowship. J.-F.R. thanks Churchill College and the French Embassy in the UK for supporting his stay at the Cavendish Laboratory.

\section{APPENDIX A: SIMULATION OF THE NV PHOTODYNAMICS}

To capture the photodynamics of the NV center, we use a seven-state model that includes the ground-state and excited-state fine structures of the NV (Fig. 6). The strain splitting is $E_{\mathrm{GS}}=E_{\mathrm{ES}} \approx 0$, where the subscripts "GS" and "ES" indicate the optical ground state and the optical excited state, respectively. At zero field, the levels $|i\rangle$ with $i=0,1,2$ are split by $D_{\mathrm{GS}}=2.87 \mathrm{GHz}$ in the optical ground state, while the levels of the excited state $|i\rangle$, $i=3,4,5$, are split by the excited state zero-field splitting $D_{\mathrm{ES}}=1.42 \mathrm{GHz}$. The transition rates from level $|i\rangle$ to the level $|j\rangle$ are denoted as $\gamma_{i j}$. The decay rates are defined as in the work by Tetienne et al. [12]: we assume the $\gamma_{30}=$ $\gamma_{41}=\gamma_{52}=\gamma_{r}, \gamma_{46}=\gamma_{56}$, and $\gamma_{61}=\gamma_{62}$. The spin nonconserving transitions from the excited state are assumed to be forbidden. Optical excitation pumps the ground-state populations to the excited state but stimulated emission is neglected, the laser being off-resonant and the vibrational relaxation decay time being short. The values used for the
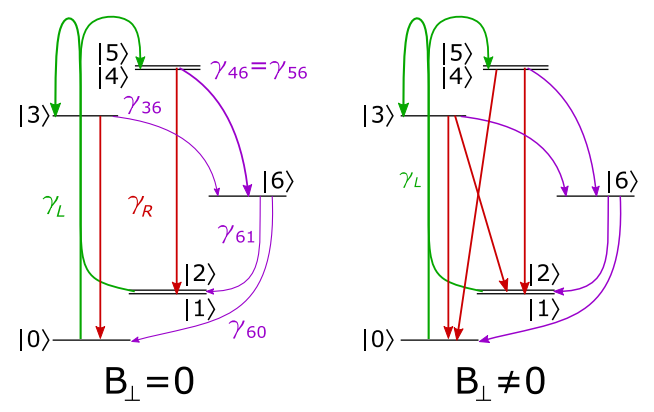

FIG. 6. Schematics of the NV seven-level system. Seven-level system used to capture the NV photodynamics for an arbitrary magnetic field. In general, off-axis magnetic fields couple the zero-field eigenstates and allow for spin-flip transitions that modify the zero-field photodynamics. Green lines represent laser excitation, red lines optical decay, and purple lines nonradiative decay.
TABLE I. Photodynamics parameters. The previously reported $[12,38]$ decay rates used in the seven-state model for the NV magnetic-field-dependent photodynamics.

\begin{tabular}{lc}
\hline \hline & Decay rate $(\mathrm{MHz})$ \\
\hline$\gamma_{r}$ & 65 \\
$\gamma_{36}$ & 11 \\
$\gamma_{46}=\gamma_{56}$ & 80 \\
$\gamma_{60}=\gamma_{62}$ & 3 \\
$\gamma_{61}=\gamma_{62}$ & 3 \\
\hline \hline
\end{tabular}

numerical simulations are taken from the works of Robledo et al. [38] and Tetienne et al. [12] (Table I). Within the assumption of Markovian noise,

$$
\begin{aligned}
\frac{d \rho(t)}{d t}= & -\frac{i}{\hbar}[\mathscr{H}, \rho]-\frac{1}{2} \sum_{k=0}^{m}\left(L_{k}^{\dagger} L_{k} \rho+\rho L_{k}^{\dagger} L_{k}\right) \\
& +\sum_{k=0}^{m} L_{k} \rho L_{k}^{\dagger},
\end{aligned}
$$

where $\mathscr{H}$ is the magnetic-field-dependent Hamiltonian describing the seven-state system, $\rho$ is the density operator, and the $L_{k}$ are the Kraus operators that describe the $m$ photon emission or absorption processes. We work in the approximation of a weaker microwave excitation rate than the laser pumping; hence, $T_{2}^{*}$ dephasing is neglected $[12,38]$. The laser pump is described as an incoherent absorption process. The Kraus operators can then either take the form

$$
L_{k}^{\mathrm{abs}}=\sqrt{\gamma_{j i}}|i\rangle\langle j|, \quad i=(3,4,5), j=(0,1,2),
$$

or

$$
L_{k}^{\mathrm{em}}=\sqrt{\gamma_{i j}}|j\rangle\langle i|, \quad i=(3,4,5,6), j=(0,1,2) .
$$

Extra Kraus operators can be added if incoherent microwave driving is included in the model:

$$
L_{k}^{\mathrm{MW}}=\sqrt{\gamma_{i j}^{\mathrm{MW}}}|i\rangle\langle j|, \quad i, j=(0,1,2), i \neq j .
$$

The steady-state PL rate is proportional to the sum of the steady-state populations in the excited state:

$$
\Gamma_{\mathrm{PL}} \propto \sum_{i=3}^{5} \rho_{i i} .
$$

Magnetic field components orthogonal to $\hat{u}_{\mathrm{NV}}$ (off axis) couple the different spin states, modifying the branching ratio of the transitions [12] and altering the steady-state populations of the levels [Fig. 7(a)]. On the one hand, this 

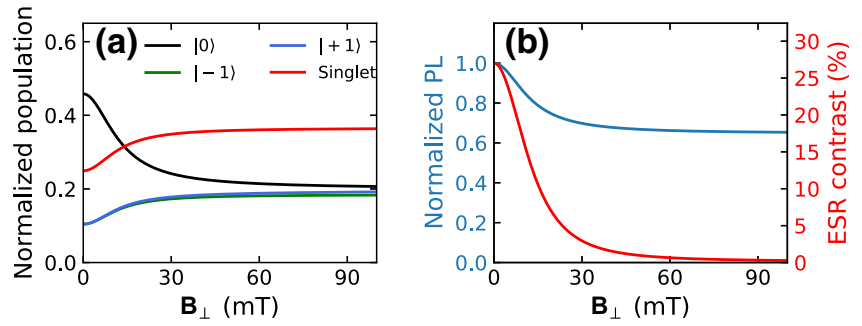

FIG. 7. Magnetic-field-dependent NV photodynamics. (a) Changes in the steady-state population under continuous green excitation of the triplet ground state and singlet state of a NV as a function of a magnetic field, $\mathbf{B}_{\perp}$, orthogonal to the $\mathrm{NV}$ axis $\hat{u}_{\mathrm{NV}}$. (b) The corresponding quench response of the NV PL (blue curve) with increasing $\mathbf{B}_{\perp}$ due to a larger shelving state population [shown in (a)]. ODMR contrast (red curve) is also reduced due to the decrease in population difference between $|0\rangle$ and $| \pm 1\rangle$ [shown in (a)].

leads to a reduction of the ODMR contrast [Fig. 7(b)], because of the reduced population difference between the $|0\rangle$ and $| \pm 1\rangle$ levels [Fig. 7(a)]. On the other hand, this leads to the quenching of the PL $[8,12,39]$, due to a larger population getting trapped in the singlet state [Fig. 7(a)]. This effect leads to a trade-off between the magnetic field amplitude $\left(\propto 1 / d_{\mathrm{NV}}\right)$ and spatial resolution $\left(\propto d_{\mathrm{NV}}\right)$ when imaging small spin textures.

\section{APPENDIX B: SNVM SETUP AND MATERIAL INFORMATION}

We perform ODMR-based NV characterization of our diamond probes and quenched-based SNVM using a magneto-optical closed-cycle helium cryostation (Attocube systems) with home-built confocal optics. Diamond probes employed are commercially procured from QZabre AG and Qnami AG. The multilayer stack of $[\operatorname{Ir}(1 \mathrm{~nm}) / \operatorname{Co}(1) / \mathrm{Pt}(1)]_{14}$ is deposited on thermally oxidized $\mathrm{Si}$ wafers by de magnetron sputtering. Additional fabrication information is found in previous studies [34]. Relevant properties of the $\mathrm{Ir} / \mathrm{Co} / \mathrm{Pt}$ stack are shown in Table II. The surface magnetization $I_{s}$ is given by $M_{s} t_{\mathrm{eff}}$, where $t_{\text {eff }}$ is the effective magnetic thickness that is the number of repetitions multiplied by the thickness of the magnetic layer. In this case, $t_{\mathrm{eff}}=14 \mathrm{~nm}$, and hence

TABLE II. Material properties. The saturation magnetization $M_{s}$, effective anisotropy $K_{\text {eff }}$, and DMI strength $D$ of the $[\mathrm{Ir} / \mathrm{Co} / \mathrm{Pt}]_{14}$ film.

\begin{tabular}{ccc}
\hline \hline $\begin{array}{c}M_{s} \\
(\mathrm{MA} / \mathrm{m})\end{array}$ & $\begin{array}{c}K_{\mathrm{eff}} \\
\left(\mathrm{MJ} / \mathrm{m}^{3}\right)\end{array}$ & $\begin{array}{c}D \\
\left(\mathrm{~mJ} / \mathrm{m}^{2}\right)\end{array}$ \\
\hline 0.881 & 0.474 & 1.25 \\
\hline \hline
\end{tabular}

TABLE III. Material systems. The surface magnetization $I_{s}$ of various systems studied with quenched SNVM compared to $[\mathrm{Ir} / \mathrm{Co} / \mathrm{Pt}]_{14}$.

\begin{tabular}{lc}
\hline \hline Material system & $I_{s}(\mathrm{~mA})$ \\
\hline $14 \times \mathrm{Ir} / \mathrm{Co} / \mathrm{Pt}$ & 12.3 \\
$\mathrm{Pt} / \mathrm{CFA} / \mathrm{MgO} / \mathrm{Ta}[22]$ & 1.8 \\
$\mathrm{CFA}: \mathrm{Co}{ }_{2} \mathrm{FeAl}$ & \\
$\mathrm{Pt} / \mathrm{FM} / \mathrm{Au} / \mathrm{FM} / \mathrm{Pt}[21]$ & 2.6 \\
$\mathrm{FM}: \mathrm{Ni} / \mathrm{Co} / \mathrm{Ni}$ & 1.7 \\
$\mathrm{Pt} / \mathrm{Co} / \mathrm{NiFe} / \mathrm{IrMn}[23]$ & \\
\hline \hline
\end{tabular}

$I_{S}=12.3 \mathrm{~mA}$ (Table. III). The $I_{S}$ of various systems studied with quenched SNVM is given in Table III for comparison. The zero-field magnetic domains are stabilized by demagnetizing the sample. This results in labyrinth morphology with a period $P=407 \mathrm{~nm}$ [Fig. 10(a)]. The sample is marked with a wirebonder (Fig. 8) that allows us to image the same area of interest (yellow box in Fig. 8) using two techniques (SNVM and MFM) on separate platforms. MFM is always carried out before and after quenched SNVM, to ensure that the morphology of the probed area remains identifiable and the features are largely unchanged.

\section{APPENDIX C: MICROMAGNETIC SIMULATIONS}

The magnetic field above the spin texture is obtained via MUMAX ${ }^{3}$ simulations. For the study of quenched imaging in various regimes (see Fig. 2 of the main text), the multilayer film is modeled using the effective medium method [40], so as to reduce computation resources. The simulation grid consists of $256 \times 256 \times 128$ cells spanning $10 \mu \mathrm{m} \times 10 \mu \mathrm{m} \times 384 \mathrm{~nm}$ (cell size is approximately $39 \times 39 \times 3 \mathrm{~nm}^{3}$ ). The first 14 layers are modeled with an effective saturation magnetization $M_{\text {eff }}=M_{s} / 3$ and the volume above as nonmagnetic spacers.

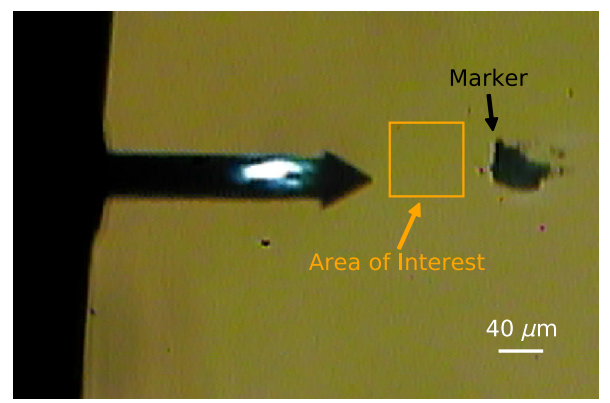

FIG. 8. Marked sample. Microscopic image of a marked area of the sample surface with a MFM probe in view. The marking is achieved using a wirebonding tip, and the area of interest probed by quenched SNVM, micromagnetics, and MFM in the main text is highlighted in yellow. 

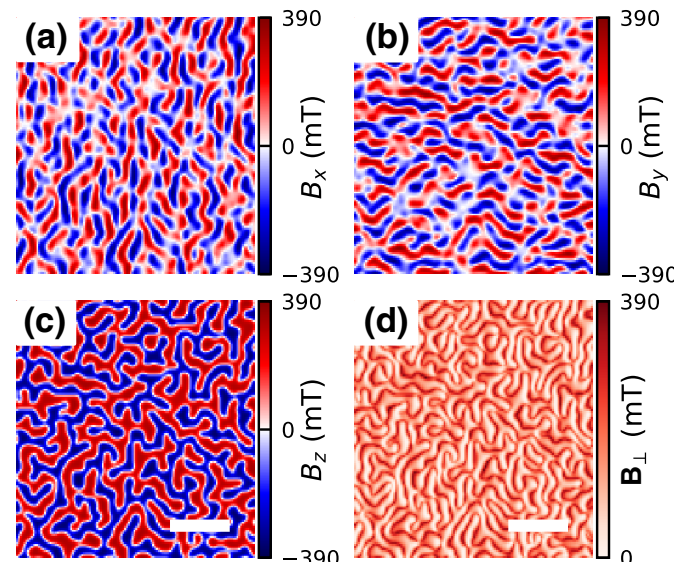

(d)

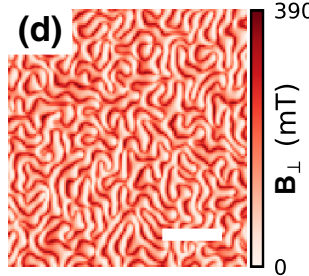

FIG. 9. Simulated magnetic field. (a)-(d) Magnetic field components $B_{x}, B_{y}$, and $B_{z}$, and the component perpendicular to the $\mathrm{NV}$ axis $\mathbf{B}_{\perp \mathrm{NV}}$ at $d_{\mathrm{NV}}=77 \mathrm{~nm}$ above the sample surface, simulated based on the magnetization distribution in Fig. 10(b). (Scale bar is $1 \mu \mathrm{m}$.)

The simulation is further refined for comparison with experiments (Figs. 3 and 4 of the main text) with each cell layer corresponding to $1 \mathrm{~nm}$ of Ir, Co, or Pt. Maintaining the same grid size, this reduces the total simulated height to $128 \mathrm{~nm}$. Similarly, the Pt, Ir layer and the volume above the multilayer film are modeled as nonmagnetic spacers. Differing from the effective medium model, the Co layer has the experimentally obtained magnetization $M_{s}$. In both cases, the simulated nonmagnetic volume above the multilayer film allows us to retrieve the magnetostatic field environment above the spin texture (Fig. 9) via MUMAX ${ }^{3}$. The magnetization distribution used in the simulation is based on segmenting a MFM image into up and down domains by image thresholding (Fig. 10).

\section{APPENDIX D: ANALYSIS OF QUENCHED IMAGING REGIMES}

The diagram in Fig. 2 of the main text is constructed based on the directionality of the observed PL features and the contrast of quenched images with different

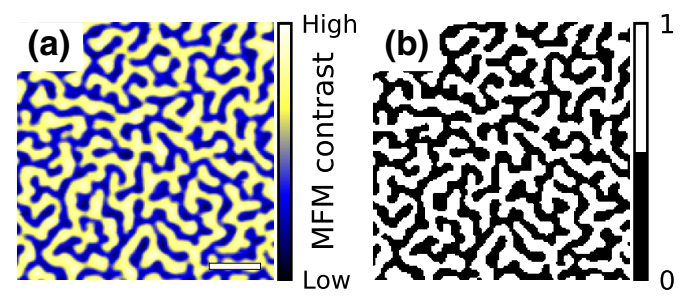

FIG. 10. Image thresholding. (a) MFM image of the sample surface (highlighted in Fig. 8), showing labyrinth domains at zero field. (b) Corresponding binary image after the thresholding process, yielding the up and down magnetization used for the simulations in Fig. 9. (Scale bar is $1 \mu \mathrm{m}$.)
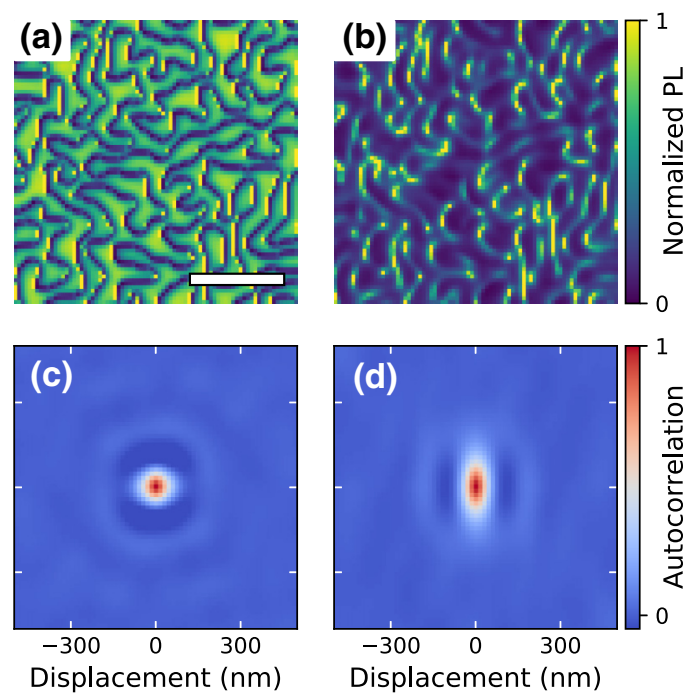

FIG. 11. Quenched image autocorrelation and directionality. (a) Quenched images at $d_{\mathrm{NV}}=12 \mathrm{~nm}$ and $I_{s}=1.6 \mathrm{~mA}$ and (b) at $d_{\mathrm{NV}}=78 \mathrm{~nm}$ and $I_{s}=10.5 \mathrm{~mA}$ (scale bar is $1 \mu \mathrm{m}$ ). (c),(d) Autocorrelation maps of panels (a) and (b), respectively. Quenched maps with low directionality display an autocorrelation peak with circular symmetry. When the directionality increases, the peak becomes elliptic.

combinations of surface magnetization $I_{S}$ and NV-sample distance $d_{\mathrm{NV}}$. The directionality of the PL features is determined from the autocorrelation of the quenched image (Fig. 11). The directionality is defined as $1-r_{\min } / r_{\max }$, where $r_{\min }$ and $r_{\max }$ are respectively the minor and major axes of an elliptical Gaussian fit to the autocorrelation peak. A directionality equal to zero indicates isotropic features [Fig. 12(a)], and a value increasing to unity implies increasing anisotropy. The PL contrast is given as
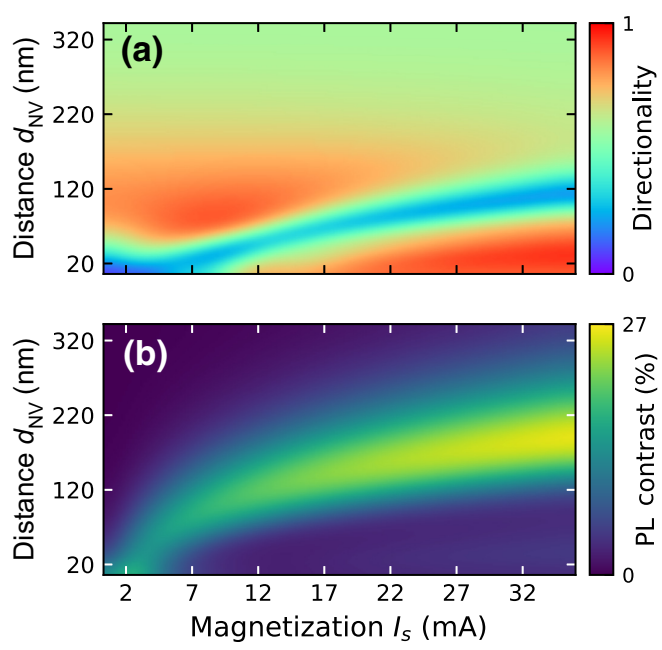

FIG. 12. Details on quenched imaging regimes. (a) The directionality of PL features and (b) the PL contrast of a quenched image given as a function of $d_{\mathrm{NV}}$ and $I_{s}$. 
$1-\mathrm{PL}_{10} / \mathrm{PL}_{90}$, where $\mathrm{PL}_{x}$ is the $x$ th percentile of the $\mathrm{PL}$ distribution of each quenched image [Fig. 12(b)].

Apart from the surface magnetization $I_{s}$ and NV-sample distance $d_{\mathrm{NV}}$ we expect the magnetic field distribution to be heavily influenced by the domain periodicity $P$. We show here that the quench imaging regimes put forward in the main paper remain valid at different $P$ with appropriate scaling of $d_{\mathrm{NV}}$ and $I_{s}$. We defined the scaled $d_{\mathrm{NV}}, d_{\mathrm{NV}}$, and scaled $I_{s}, I_{s}^{\prime}$ as

$$
\begin{aligned}
& d_{\mathrm{NV}}(P)=d_{\mathrm{NV}} \times C_{d}(P)=d_{\mathrm{NV}} \times\left(P / P_{0}\right)^{S_{d}}, \\
& I_{s}^{\prime}(P)=I_{s} / I_{s, 0} \times C_{i}(P)=I_{s} / I_{s, 0} \times\left(P / P_{0}\right)^{S_{i}},
\end{aligned}
$$

where $C_{d}$ and $C_{i}$ are the scaling factors, $S_{d}$ and $S_{i}$ are the scaling exponents, while $P_{0}=400 \mathrm{~nm}$ and $I_{s, 0}=$
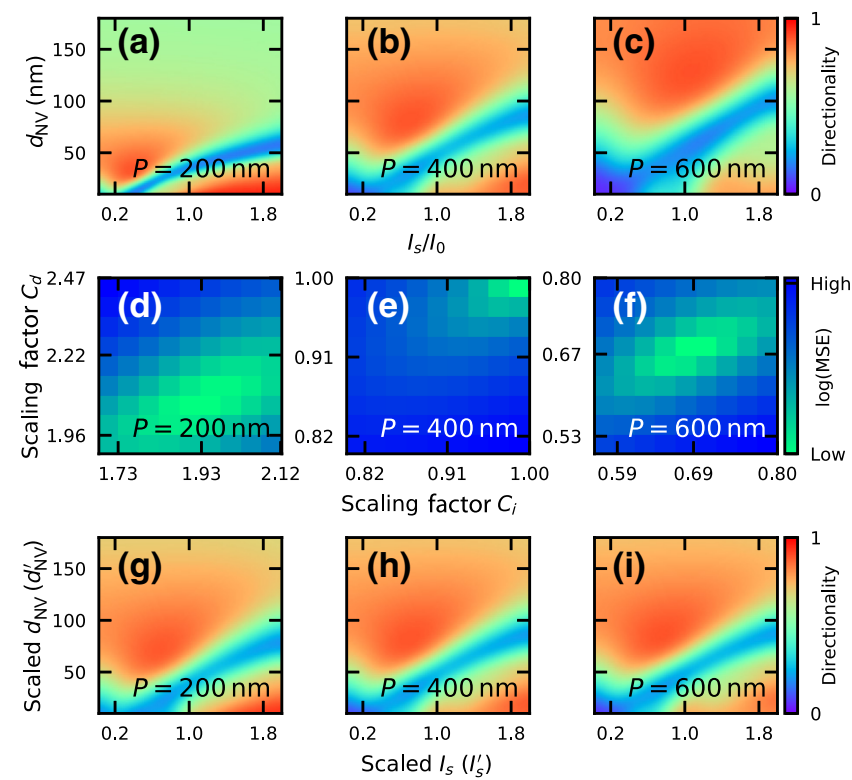

FIG. 13. Quenched imaging regimes at varying domain periodicities and associated scalings. (a)-(c) The directionality of PL features as a function of the NV-sample distance $d_{\mathrm{NV}}$ and surface magnetization $I_{s}$ at domain periodicities (a) $P=200 \mathrm{~nm}$, (b) $P=400 \mathrm{~nm}$, and (c) $P=600 \mathrm{~nm}$. (d) -(f) A range of scaling factors $C_{d}, C_{i}$ are applied to the directional plots at various $P$ (a)-(c) and the MSE with reference to the unscaled plot at $P=400 \mathrm{~nm}$ (b) are given in panels (d)-(f). The optimal $C_{d}, C_{i}$ pair is estimated by the minimum MSE. Note that, for $P=400 \mathrm{~nm}$ (e), the optimal scaling factor of $C_{d}, C_{i}=1$ has a $\log (\mathrm{MSE})$ value of negative infinity since the expected MSE is zero. (g)-(i) Scaled directional plots at various $P$ after applying the optimal scaling factors $C_{d}, C_{i}$ determined by the minimum MSE given in (d)-(f). The similarity observed across the scaled directionality plots (g)-(i) indicates that the quench imaging regimes remain valid across different $P$ with appropriate scaling to $I_{S}$ and $d_{\mathrm{NV}}$. (a)

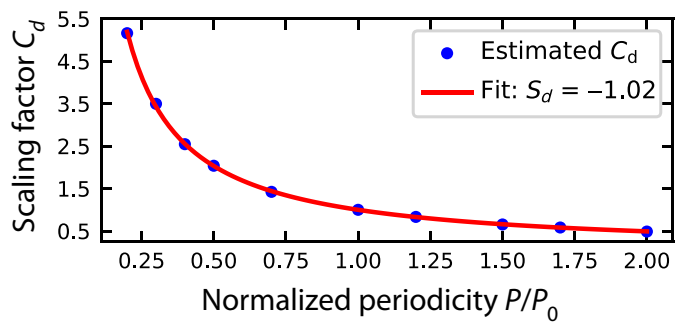

(b)

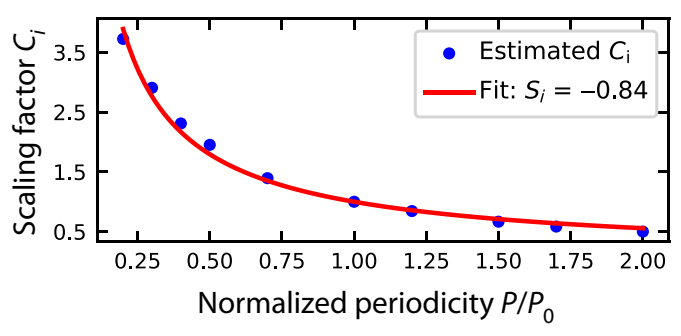

FIG. 14. Estimation of the scaling exponents. (a),(b) Fitting of scaling equations [Eqs. (D1) and (D2)] to the scaling factors (a) $C_{d}$ and (b) $C_{i}$ (see Fig. 13 for estimation details) as a function of the domain periodicity $P$. The reference $P, P_{0}$, is the periodicity observed experimentally at $400 \mathrm{~nm}$. The scaling exponents $S_{d}$ and $S_{i}$ (associated with $d_{\mathrm{NV}}$ and $I_{s}$ ) have estimated values of -1 and -0.8 .

$12.3 \mathrm{~mA}$, corresponding to the values for our sample $[\operatorname{Ir}(1 \mathrm{~nm}) / \operatorname{Co}(1) / \operatorname{Pt}(1)]_{14}$. The scaling factors $C_{d}(P)$ and $C_{i}(P)$ as a function of periodicity $P(80,120,160,200$, $280,480,600,680,800 \mathrm{~nm})$ are estimated by first scaling the respective directional plots [Figs. 13(a)-13(c)] and performing an image registration to the reference directional plot at $P=400 \mathrm{~nm}$ [Fig. 13(b)]. For each $P$, ranges of $C_{d}$ and $C_{i}$ are applied to the directional plot and registered to the reference. The mean square error (MSE) between the scaled and reference directionality plots is then computed for each $C_{d}, C_{i}$ pair [Figs. 13(d)-13(f)]. The optimal $C_{d}, C_{i}$ pair at each $P$ is determined from the minimum MSE. The estimated $C_{d}(P)$ and $C_{i}(P)$ are collated and fitted to Eqs. (D1) and (D2) to retrieve the scaling exponents, $S_{d}=-1$ and $S_{i}=-0.8$ [Figs. 14(a) and 14(b)]. The scaled directional plots at various $P$ are given in Figs. 13(g)-13(i). The similarity observed across the scaled directionality plots [Figs. 13(g)-13(i)] indicates that the quench imaging regimes remain valid across different $P$ with appropriate scaling given by Eqs. (D1) and (D2). We have also included films studied by Gross et al. [21] and Rana et al. [23] in this framework (Fig. 15). The framework is in good agreement with the work of Gross et al., who observed isotropic PL features. In the study of Rana et al., we are unable to resolve the directionality of the features observed. However, we expect the quenched imaging regime to deviate from our framework as our simulation model does not include exchange bias present in their film. 


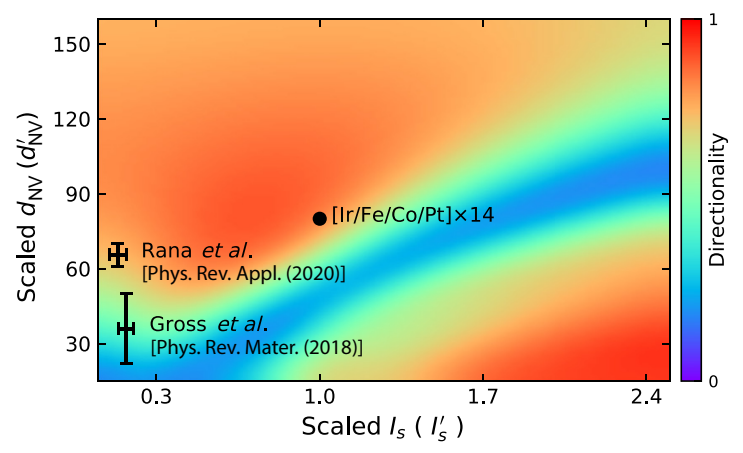

FIG. 15. Overview of quenched imaging on thin films. Previous studies involving quenched imaging of thin films are plotted on the scaled directionality map. The position on the map is based on the $d_{\mathrm{NV}}, I_{s}$, and $P$ in each study.

\section{APPENDIX E: NV SENSOR CHARACTERIZATION}

We use a three-axis Helmholtz coil to apply an external magnetic field $\mathbf{B}$ at varying $\varphi$ and $\vartheta$, with a fixed field strength $|\mathbf{B}|=1 \mathrm{mT}$. We obtain the ODMR spectra by recording the integrated PL intensity of the NV center as we sweep the microwave (MW) frequency. In the presence of a magnetic field, the ODMR spectrum displays a splitting of the $\left|m_{s}=+1\right\rangle$ and $\left|m_{s}=-1\right\rangle$ states due to the Zeeman effect [Fig. 16(a)]. This splitting is proportional to the projection of the magnetic field on the NV axis $\hat{u}_{\mathrm{NV}}$. The ODMR spectrum is first obtained as a function of $\varphi$ while fixing $\vartheta=90^{\circ}$ [Fig. 16(b)]. The Zeeman splitting is maximum when $\varphi=\varphi_{\mathrm{NV}}$, which in our case is $\varphi_{\mathrm{NV}}=93^{\circ} \pm 2^{\circ}$. Next, we vary $\vartheta$ while fixing $\varphi=\varphi_{\mathrm{NV}}$ [Fig. 16(c)]. Similarly, the maximum splitting occurs when $\vartheta=\vartheta_{\mathrm{NV}}$, which we obtain to be $\vartheta_{\mathrm{NV}}=60^{\circ} \pm 2^{\circ}$.

We determine the NV-sample distance $d_{\mathrm{NV}}$ by measuring with our diamond tip the stray field emitted across the edge of a $[\mathrm{Ta} / \mathrm{Co}-\mathrm{Fe}-\mathrm{B} / \mathrm{MgO}]$ strip. The out-of-plane magnetic hysteresis is characterized by a MagVision (a)

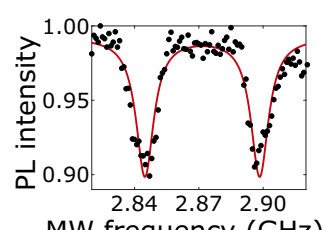

$\mathrm{MW}$ frequency $(\mathrm{GHz})$ (b)

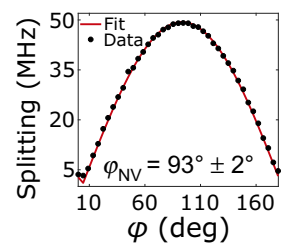

(a)

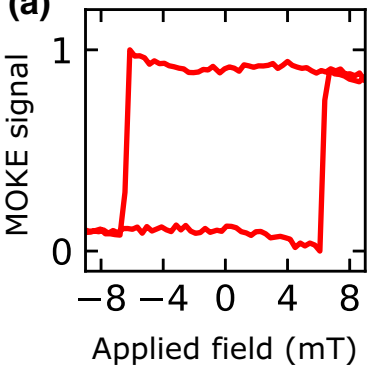

(b)

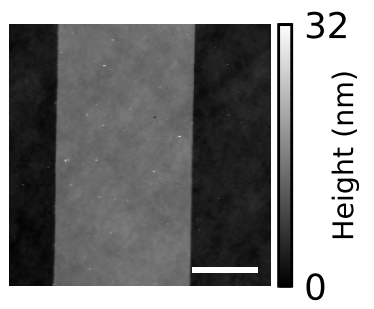

FIG. 17. Calibration strip characterization. (a) Intensity of the polar MOKE signal of a $[\mathrm{Ta} / \mathrm{Co}-\mathrm{Fe}-\mathrm{B} / \mathrm{MgO}]$ strip as a function of an out-of-plane magnetic field. (b) Topography image of the $[\mathrm{Ta} / \mathrm{Co}-\mathrm{Fe}-\mathrm{B} / \mathrm{MgO}$ ] calibration strip (scale bar is $10 \mu \mathrm{m}$ ).

magneto-optical Kerr effect (MOKE) microscope (Vertisis Technology) in the polar sensitivity mode and shows that the magnetization remains saturated at remanence (Fig. 17). The Zeeman shift of the ODMR spectrum across the edge at remanence is given in Fig. 18(a) (blue dashed curve) and is fitted (red curve) following the procedure devised by Hingant et al. [41] to retrieve $d_{\mathrm{NV}}$. We repeat the measurement numerous times along the edge at $50 \mathrm{~nm}$ spacing, and the extracted values are averaged [Fig. 18(b)]. The diamond tip used in this work has a $d_{\mathrm{NV}}=77.5 \pm$ $3 \mathrm{~nm}$.

\section{APPENDIX F: QUENCHED IMAGING WITH [111] NVs}

The discussion in the main text focuses on NVs found in commercially available (100) diamond tips. Quenched maps obtained with NVs with $\vartheta_{\mathrm{NV}}=54.7^{\circ}$ on samples with out-of-plane magnetic anisotropy give rise to different imaging regimes, as explained in the main text. Notably, there is a range of $d_{\mathrm{NV}}$ and $I_{s}$ where the quenched maps

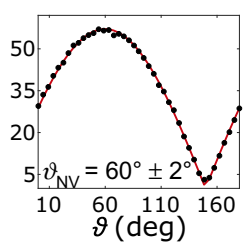

FIG. 16. Axis measurements of the NV probe. (a) ODMR spectrum obtained under an external magnetic field. We can observe the splitting of the $\left|m_{s}=+1\right\rangle$ and $\left|m_{s}=-1\right\rangle$ states due to the Zeeman effect. We measure a splitting of $54 \mathrm{MHz}$ that corresponds to a field felt by the NV of about $1 \mathrm{mT}$. (b) Measurement of $\varphi_{\mathrm{NV}}$. We fix $\vartheta$ and $\varphi$ is varying. When the ODMR splitting is maximum, $\varphi=\varphi_{\mathrm{NV}}$. (c) Measurement of $\vartheta_{\mathrm{NV}}$. We fix $\varphi$ and $\vartheta$ is varying. Here $\vartheta=\vartheta_{\mathrm{NV}}$ when the ODMR splitting reaches its maximum value. (a)

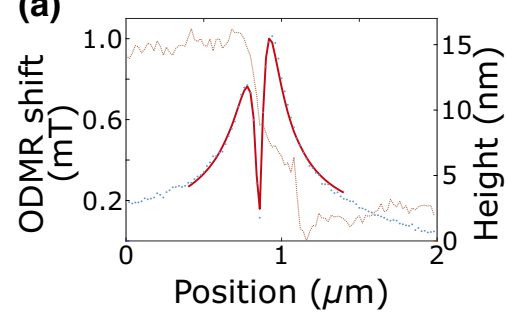

(b)

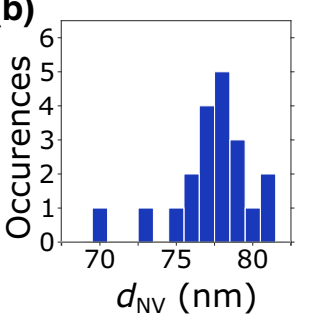

FIG. 18. Calibration of the NV probe. (a) We represent on this plot the topography of the edge of a Co-Fe-B magnetic stripe (in brown) and the measured Zeeman shift of the NV ODMR spectrum (in blue) due to the magnetic field emitted at the edge of the stripe. We deduce the value of $d_{\mathrm{NV}}$ from the fit (in red) of the Zeeman shift experimentally measured. (b) Histogram distribution of all the NV-sample distances we measured. The average value is $d_{\mathrm{NV}}=77.5 \mathrm{~nm}$ and the standard deviation is $\sigma_{d_{\mathrm{NV}}} \simeq 3 \mathrm{~nm}$. 


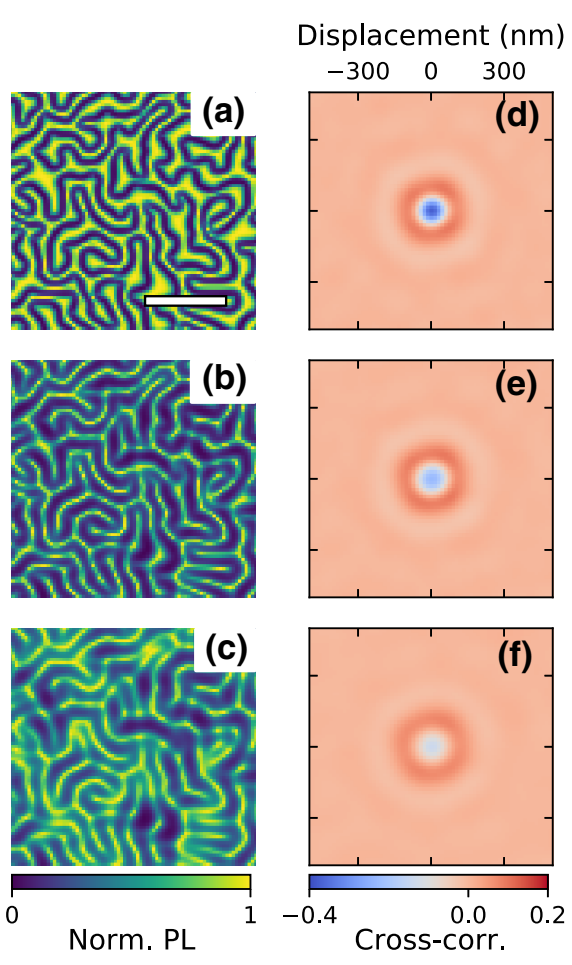

FIG. 19. Quenching with [111] NVs. Quenching maps obtained with NVs with $\vartheta_{\mathrm{NV}}=0^{\circ}$ on the same area as Fig. 2 in the main text (scale bar is $1 \mu \mathrm{m}$ ). The different maps correspond to (a) $I_{s}=3.1 \mathrm{~mA}, d_{\mathrm{NV}}=30 \mathrm{~nm}$, (b) $I_{s}=9.2 \mathrm{~mA}, d_{\mathrm{NV}}=$ $84 \mathrm{~nm}$, and (c) $I_{s}=15.4 \mathrm{~mA}, d_{\mathrm{NV}}=162 \mathrm{~nm}$, which correspond to the parameters of Figs. 2(b)-2(d) of the main text. (d)-(f) Two-dimensional cross-correlation maps between the images in (a) (c) and the domain boundaries. The negative correlation at zero displacement indicates a low PL at the boundary. If the displacement increases, the correlation is positive, corresponding to the bright PL observed within the domains.

directionally highlight the domain boundaries. The directionality is due to the nonzero angle between the NV axis and the magnetic anisotropy. Hence, this effect is not present when using NVs pointing along the [111] axis (i.e., $\vartheta_{\mathrm{NV}}=0^{\circ}$ ), hosted in [111]-oriented diamond tips, which have recently been reported [37]. The simulations are shown in Figs. 19(a)-19(c), which have been taken at the same $d_{\mathrm{NV}}$ and $I_{s}$ as Figs. 2(b)-2(d) of the main text, respectively. At low magnetization [Fig. 19(a)], the NV PL is quenched along the domain boundaries [cross-correlation in Fig. 19(d)], resulting in a bright image with dark outlines. At higher magnetization [Fig. 19(b)], the quenching still traces the domain boundaries [cross-correlation in Fig. 19(e)], but also expands further within the domain area. The thin bright lines correspond to the innermost areas of the domains, where the magnetic field is mainly orthogonal to the sample surface and thus aligned with the NV axis. In Fig. 19(c), the combination of large magnetization and high $d_{\mathrm{NV}}$ gives an image similar to Fig. 19(b), but with lower resolution.

\section{APPENDIX G: DOMAIN COVERAGE ESTIMATION}

In order to estimate the percentage of domain boundaries covered by the simulated quenched maps, we first binarize the selected MFM images with Otsu thresholding [42] [a portion is shown Fig. 20(a)] and detect the boundaries with the Canny algorithm. The quenched maps are simulated from the stray fields obtained with MUMAX ${ }^{3}$, as explained above. We first simulate the quenched maps with NVs at $\vartheta_{\mathrm{NV}}=54.7^{\circ}$ and different $\varphi_{\mathrm{NV}}$ [Fig. 20(b) for $\left.\varphi_{\mathrm{NV}}=0^{\circ}\right]$. The single images are then registered to the domain boundaries with the ECC image alignment algorithm [43], in order to compensate for the small shift from the domain boundaries induced by the nonzero angle between the magnetic anisotropy and $\vartheta_{\mathrm{NV}}$. The images are then combined as explained in the main text [Fig. 20(c) for $N=4$ and $\varphi_{\mathrm{NV}}{ }^{\max }=180^{\circ}$ ]. Additionally, we simulate a quenched map with a $\mathrm{NV}$ at $\vartheta_{\mathrm{NV}}=0^{\circ}$, which
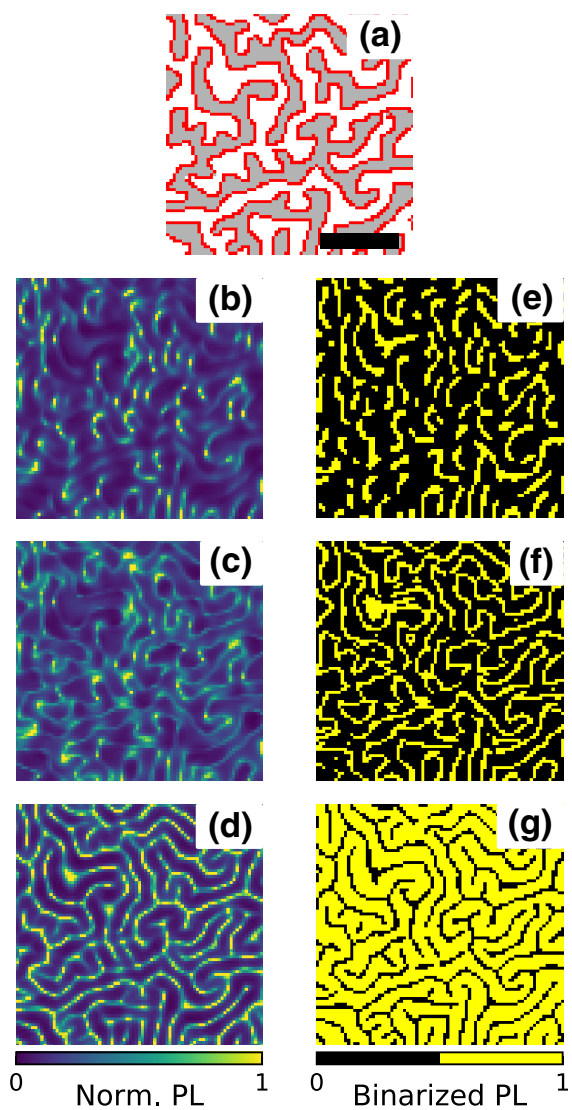

FIG. 20. Estimation of the domain coverage. (a) Portion of the binarized MFM scan (background) and domain edges (red pixels) obtained via Canny edge detection (scale bar is $1 \mu \mathrm{m}$ ). Quenched maps of the same area, where (b) displays the map taken with a $\mathrm{NV}$ at $\vartheta_{\mathrm{NV}}=54.7^{\circ}$ and $\varphi_{\mathrm{NV}}=0^{\circ}$, (c) displays the reconstructed image with $N=4$ at $\varphi_{\mathrm{NV}}{ }^{\max }=180^{\circ}$ (see the main text), and the image in (d) is acquired with a NV with $\vartheta_{\mathrm{NV}}=0^{\circ}$. Panels (e)-(g) display the images obtained by binarizing (b)-(d), respectively. 
exhibits no shift and no $\varphi_{\text {NV }}$ dependence [Fig. 20(d)]. The images are then binarized using local Gaussian thresholding [Figs. 20(e)-20(g)]. The binarized images are multiplied to the domain boundaries and integrated to yield the coverage. For experimental quenched maps, the above estimation protocol includes additional thinning and dilation of the binarized images before multiplication. The thinning and dilation process ensures that local deviations between the binarized experimental quenched maps obtained via SNVM and the domain outline retrieved from MFM images are accounted in the coverage estimation. These local deviations are largely due to experimental map nonlinearity, nonoptimal image threshold and registration conditions, and MFM perturbation. To estimate the experimental coverage error, we use the three smallest structuring element - a three-pixel-wide diamond, a threepixel-wide square, and a five-pixel-wide diamond-for binary dilation. The coverage value is given by the estimation protocol using a three-pixel-wide square dilation structuring element, while the coverage bounds are given by the diamond structuring elements.

\section{APPENDIX H: IMAGING WITH MINIMAL PERTURBATION}

As explained in the main text and in previous studies [21-23], quenched SNVM enables perturbation-free imaging of spin textures. We present here a comparison of repeated quenched SNVM and MFM scans over the same area, which highlight the nonperturbative advantage of quenched SNVM. We first obtain two consecutive quenched images over an area on the sample [Figs. 21(a) and 21(b)], and thereafter another two consecutive MFM images over the same area [Figs. 21(c) and 21(d)]. By comparing the quenched and MFM images, we observed areas [circled in Figs. 21(a)-21(e)] showing nonperturbative consecutive quenched imaging [Figs. 21(a) and 21(b)] that were subsequently perturbed by consecutive MFM scans [Figs. 21(c) and 21(d)]. In addition, the quenched image simulated [Fig. 21(e)] from the MFM image in Fig. 21(d) shows markedly different PL features compared to experiments [Figs. 21(a) and 21(b)] at the vicinity of the highlighted areas [circled in Figs. 21(a), 21(b), and 21(e)], reinforcing the nonperturbative advantage of quenched SNVM over conventional MFM. In our case, the MFM probe used for the comparison is a low moment variant from Asylum Research, Oxford Instruments (ASYMFMLM-R2). These observations are however not exhaustive in nature and require a statistical approach to determine the degree of perturbation induced by MFM over quenched SNVM. A rigorous characterization is highly nontrivial and involves a vast parameter space including various magnetic material parameters, different laser intensities utilized during quenched SNVM, and numerous low-moment probe options for MFM.

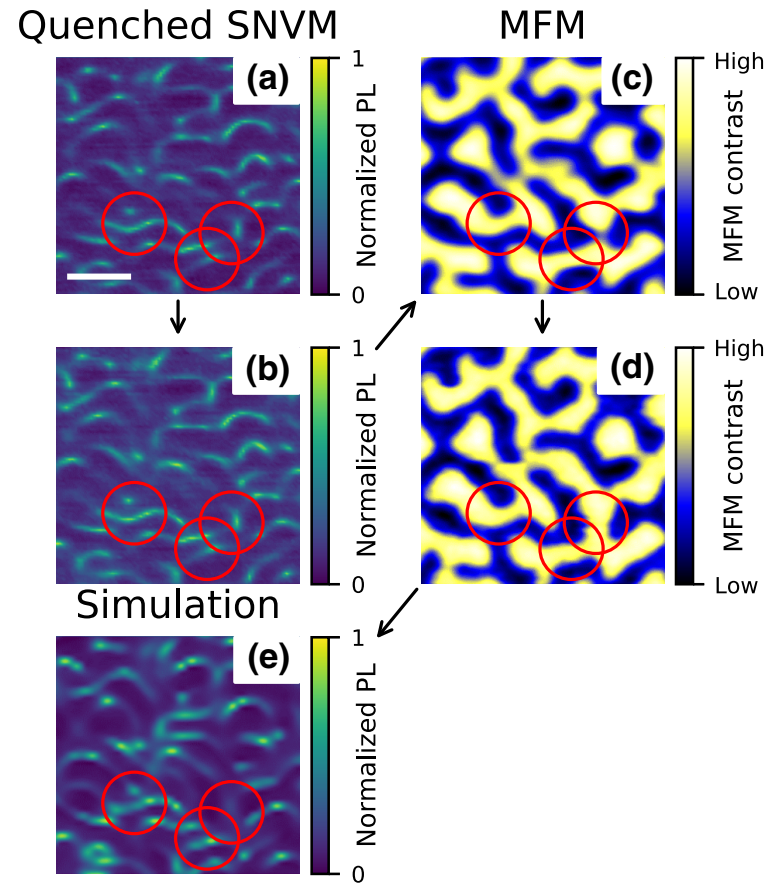

FIG. 21. Evidence of nonperturbative imaging. Sequential study of the domain morphology by (a),(b) consecutive quenched SNVM imaging, followed by (c),(d) consecutive MFM. Areas of perturbation due to consecutive MFM imaging are circled (c),(d), while no visible changes are observed in the corresponding areas in the consecutive quenched images (a),(b). (e) Simulated quenched image based on second MFM scan (d) shows dissimilar PL features in the circled vicinity as compared to experiments (a),(b) (scale bar is $500 \mathrm{~nm}$ ).

[1] G. Balasubramanian, I. Y. Chan, R. Kolesov, M. AlHmoud, J. Tisler, C. Shin, C. Kim, A. Wojcik, P. R. Hemmer, A. Krueger, T. Hanke, A. Leitenstorfer, R. Bratschitsch, F. Jelezko, and J. Wrachtrup, Nanoscale imaging magnetometry with diamond spins under ambient conditions, Nature 455, 648 (2008).

[2] P. Maletinsky, S. Hong, M. S. Grinolds, B. Hausmann, M. D. Lukin, R. L. Walsworth, M. Loncar, and A. Yacoby, A robust scanning diamond sensor for nanoscale imaging with single nitrogen-vacancy centres, Nat. Nanotechnol. 7, 320 (2012).

[3] J.-P. Tetienne, T. Hingant, J.-V. Kim, L. H. Diez, J.-P. Adam, K. Garcia, J.-F. Roch, S. Rohart, A. Thiaville, D. Ravelosona, and V. Jacques, Nanoscale imaging and control of domain-wall hopping with a nitrogen-vacancy center microscope, Science 344, 1366 (2014).

[4] I. Gross, W. Akhtar, V. Garcia, L. J. Martínez, S. Chouaieb, K. Garcia, C. Carrétéro, A. Barthélémy, P. Appel, P. Maletinsky, J.-V. Kim, J. Y. Chauleau, N. Jaouen, M. Viret, M. Bibes, S. Fusil, and V. Jacques, Real-space imaging of non-collinear antiferromagnetic order with a single-spin magnetometer, Nature 549, 252 (2017). 
[5] L. Thiel, Z. Wang, M. A. Tschudin, D. Rohner, I. GutiérrezLezama, N. Ubrig, M. Gibertini, E. Giannini, A. F. Morpurgo, and P. Maletinsky, Probing magnetism in 2D materials at the nanoscale with single-spin microscopy, Science 364, 973 (2019).

[6] L. Rondin, J.-P. Tetienne, T. Hingant, J.-F. Roch, P. Maletinsky, and V. Jacques, Magnetometry with nitrogenvacancy defects in diamond, Rep. Prog. Phys. 77, 056503 (2014).

[7] J.-P. Tetienne, T. Hingant, L. J. Martínez, S. Rohart, A. Thiaville, L. H. Diez, K. Garcia, J.-P. Adam, J.-V. Kim, J.-F. Roch, I. M. Miron, G. Gaudin, L. Vila, B. Ocker, D. Ravelosona, and V. Jacques, The nature of domain walls in ultrathin ferromagnets revealed by scanning nanomagnetometry, Nat. Commun. 6, 6733 (2015).

[8] L. Rondin, J.-P. Tetienne, P. Spinicelli, C. Dal Savio, K. Karrai, G. Dantelle, A. Thiaville, S. Rohart, J.-F. Roch, and $\mathrm{V}$. Jacques, Nanoscale magnetic field mapping with a single spin scanning probe magnetometer, Appl. Phys. Lett. 100, 153118 (2012).

[9] P. Appel, E. Neu, M. Ganzhorn, A. Barfuss, M. Batzer, M. Gratz, A. Tschöpe, and P. Maletinsky, Fabrication of all diamond scanning probes for nanoscale magnetometry, Rev. Sci. Instrum. 87, 063703 (2016).

[10] C. Degen, Microscopy with single spins, Nat. Nanotechnol. 3, 643 (2008).

[11] Y. Dovzhenko, F. Casola, S. Schlotter, T. X. Zhou, F. Büttner, R. L. Walsworth, G. S. D. Beach, and A. Yacoby, Magnetostatic twists in room-temperature skyrmions explored by nitrogen-vacancy center spin texture reconstruction, Nat. Commun. 9, 2712 (2018).

[12] J. P. Tetienne, L. Rondin, P. Spinicelli, M. Chipaux, T. Debuisschert, J. F. Roch, and V. Jacques, Magnetic-fielddependent photodynamics of single NV defects in diamond: An application to qualitative all-optical magnetic imaging, New J. Phys. 14, 103033 (2012).

[13] J.-P. Tetienne, T. Hingant, L. Rondin, S. Rohart, A. Thiaville, E. Jué, G. Gaudin, J.-F. Roch, and V. Jacques, Nitrogen-vacancy-center imaging of bubble domains in a 6$\AA$ film of cobalt with perpendicular magnetization, J. Appl. Phys. 115, 17D501 (2014).

[14] T. Kosub, M. Kopte, R. Hühne, P. Appel, B. Shields, P. Maletinsky, R. Hübner, M. O. Liedke, J. Fassbender, O. G. Schmidt, and D. Makarov, Purely antiferromagnetic magnetoelectric random access memory, Nat. Commun. 8, 13985 (2017).

[15] M. S. Wörnle, P. Welter, Z. Kašpar, K. Olejník, V. Novák, R. P. Campion, P. Wadley, T. Jungwirth, C. L. Degen, and P. Gambardella, Current-induced fragmentation of antiferromagnetic domains, arXiv:1912.05287 (2019).

[16] A. Jenkins, M. Pelliccione, G. Yu, X. Ma, X. Li, K. L. Wang, and A. C. B. Jayich, Single spin sensing of domain wall structure and dynamics in a thin film skyrmion host, Phys. Rev. Mater. 3, 083801 (2019).

[17] P. Appel, B. J. Shields, T. Kosub, N. Hedrich, R. Hübner, J. Faßbender, D. Makarov, and P. Maletinsky, Nanomagnetism of magnetoelectric granular thin-film antiferromagnets, Nano Lett. 19, 1682 (2019).

[18] Q.-C. Sun, T. Song, E. Anderson, A. Brunner, J. Förster, T. Shalomayeva, T. Taniguchi, K. Watanabe, J. Gräfe, R.
Stöhr, X. Xu, and J. Wrachtrup, Magnetic domains and domain wall pinning in atomically thin $\mathrm{CrBr} 3$ revealed by nanoscale imaging, Nat. Commun. 12, 1989 (2021).

[19] N. Hedrich, K. Wagner, O. V. Pylypovskyi, B. J. Shields, T. Kosub, D. D. Sheka, D. Makarov, and P. Maletinsky, Nanoscale mechanics of antiferromagnetic domain walls, Nat. Phys. 17, 574 (2021).

[20] M. S. Wörnle, P. Welter, M. Giraldo, T. Lottermoser, M. Fiebig, P. Gambardella, and C. L. Degen, Coexistence of Bloch and Néel walls in a collinear antiferromagnet, Phys. Rev. B 103, 094426 (2021).

[21] I. Gross, W. Akhtar, A. Hrabec, J. Sampaio, L. J. Martínez, S. Chouaieb, B. J. Shields, P. Maletinsky, A. Thiaville, S. Rohart, and V. Jacques, Skyrmion morphology in ultrathin magnetic films, Phys. Rev. Mater. 2, 024406 (2018)

[22] W. Akhtar, A. Hrabec, S. Chouaieb, A. Haykal, I. Gross, M. Belmeguenai, M. Gabor, B. Shields, P. Maletinsky, A. Thiaville, S. Rohart, and V. Jacques, Current-Induced Nucleation and Dynamics of Skyrmions in a Co-Based Heusler Alloy, Phys. Rev. Appl. 11, 034066 (2019).

[23] K. G. Rana, A. Finco, F. Fabre, S. Chouaieb, A. Haykal, L. D. Buda-Prejbeanu, O. Fruchart, S. Le Denmat, P. David, M. Belmeguenai, T. Denneulin, R. E. Dunin-Borkowski, G. Gaudin, V. Jacques, and O. Boulle, Room-Temperature Skyrmions at Zero Field in Exchange-Biased Ultrathin Films, Phys. Rev. Appl. 13, 044079 (2020).

[24] A. Wickenbrock, H. Zheng, L. Bougas, N. Leefer, S. Afach, A. Jarmola, V. M. Acosta, and D. Budker, Microwave-free magnetometry with nitrogen-vacancy centers in diamond, Appl. Phys. Lett. 109, 053505 (2016).

[25] H. Zheng, Z. Sun, G. Chatzidrosos, C. Zhang, K. Nakamura, H. Sumiya, T. Ohshima, J. Isoya, J. Wrachtrup, A. Wickenbrock, and D. Budker, Microwave-Free Vector Magnetometry with Nitrogen-Vacancy Centers along a Single Axis in Diamond, Phys. Rev. Appl. 13, 044023 (2020).

[26] A. Finco, A. Haykal, R. Tanos, F. Fabre, S. Chouaieb, W. Akhtar, I. Robert-Philip, W. Legrand, F. Ajejas, K. Bouzehouane, N. Reyren, T. Devolder, J.-P. Adam, J.-V. Kim, V. Cros, and V. Jacques, Imaging non-collinear antiferromagnetic textures via single spin relaxometry, Nat. Commun. 12, 767 (2021).

[27] T. Van Der Sar, F. Casola, R. Walsworth, and A. Yacoby, Nanometre-scale probing of spin waves using singleelectron spins, Nat. Commun. 6, 7886 (2015).

[28] T. X. Zhou, R. J. Stöhr, and A. Yacoby, Scanning diamond nv center probes compatible with conventional afm technology, Appl. Phys. Lett. 111, 163106 (2017).

[29] M. W. Doherty, F. Dolde, H. Fedder, F. Jelezko, J. Wrachtrup, N. B. Manson, and L. C. L. Hollenberg, Theory of the ground-state spin of the NV-center in diamond, Phys. Rev. B 85, 205203 (2012).

[30] M. W. Doherty, N. B. Manson, P. Delaney, F. Jelezko, J. Wrachtrup, and L. C. Hollenberg, The nitrogen-vacancy colour centre in diamond, Phys. Rep. 528, 1 (2013).

[31] M. W. Doherty, N. B. Manson, P. Delaney, and L. C. L. Hollenberg, The negatively charged nitrogen-vacancy centre in 
diamond: The electronic solution, New. J. Phys. 13, 025019 (2011).

[32] A. Gruber, A. Dräbenstedt, C. Tietz, L. Fleury, J. Wrachtrup, and C. v. Borczyskowski, Scanning confocal optical microscopy and magnetic resonance on single defect centers, Science 276, 2012 (1997).

[33] C. Moreau-Luchaire, C. Moutafis, N. Reyren, J. Sampaio, C. A. F. Vaz, N. Van Horne, K. Bouzehouane, K. Garcia, C. Deranlot, P. Warnicke, P. Wohlhüter, J.-M. George, M. Weigand, J. Raabe, V. Cros, and A. Fert, Additive interfacial chiral interaction in multilayers for stabilization of small individual skyrmions at room temperature, Nat. Nanotechnol. 11, 444 (2016).

[34] A. Soumyanarayanan, M. Raju, A. L. Gonzalez Oyarce, A. K. C. Tan, M.-Y. Im, A. P. Petrović, P. Ho, K. H. Khoo, M. Tran, C. K. Gan, F. Ernult, and C. Panagopoulos, Tunable room-temperature magnetic skyrmions in $\mathrm{Ir} / \mathrm{Fe} / \mathrm{Co} / \mathrm{Pt}$ multilayers, Nat. Mater. 16, 898 (2017).

[35] O. Kazakova, R. Puttock, C. Barton, H. Corte-León, M. Jaafar, V. Neu, and A. Asenjo, Frontiers of magnetic force microscopy, J. Appl. Phys. 125, 060901 (2019).

[36] A. Vansteenkiste, J. Leliaert, M. Dvornik, M. Helsen, F. Garcia-Sanchez, and B. Van Waeyenberge, The design and verification of mumax3, AIP Adv. 4, 107133 (2014).

[37] D. Rohner, J. Happacher, P. Reiser, M. A. Tschudin, A. Tallaire, J. Achard, B. J. Shields, and P. Maletinsky, (111)-oriented, single crystal diamond tips for nanoscale scanning probe imaging of out-of-plane magnetic fields, Appl. Phys. Lett. 115, 192401 (2019).

[38] L. Robledo, H. Bernien, T. van der Sar, and R. Hanson, Spin dynamics in the optical cycle of single nitrogenvacancy centres in diamond, New J. Phys. 13, 025013 (2011).

[39] L. Stefan, Ph.D. thesis, University of Bristol (2020).

[40] S. Woo, K. Litzius, B. Krüger, M.-Y. Im, L. Caretta, K. Richter, M. Mann, A. Krone, R. M. Reeve, M. Weigand, P. Agrawal, I. Lemesh, M.-A. Mawass, P. Fischer, M. Kläui, and G. S. D. Beach, Observation of room-temperature magnetic skyrmions and their current-driven dynamics in ultrathin metallic ferromagnets, Nat. Mater. 15, 501 (2016).

[41] T. Hingant, J.-P. Tetienne, L. J. Martínez, K. Garcia, D. Ravelosona, J.-F. Roch, and V. Jacques, Measuring the Magnetic Moment Density in Patterned Ultrathin Ferromagnets with Submicrometer Resolution, Phys. Rev. Appl. 4, 014003 (2015).

[42] N. Otsu, A threshold selection method from graylevel histograms, IEEE Trans. Syst. Man Cybern. 9, 62 (1979).

[43] G. D. Evangelidis and E. Z. Psarakis, Parametric image alignment using enhanced correlation coefficient maximization, IEEE Trans. Pattern Anal. Mach. Intell. 30, 1858 (2008). 